\title{
A REVIEW ON HUMAN-EXOSKELETON COORDINATION TOWARDS LOWER LIMB ROBOTIC EXOSKELETON SYSTEMS
}

\author{
Yue Ma, ${ }^{*, * *}$ Xinyu $\mathrm{Wu},{ }^{*, * * *}$ Jingang $\mathrm{Yi},{ }^{* * * *}$ Can Wang, ${ }^{*}$ and Chunjie Chen ${ }^{*}$
}

\begin{abstract}
The study of lower extremity exoskeleton robots has been pursued for many years and is now receiving significant attention. A number of review articles have focused on describing the mechanical design of exoskeleton robots, their control methods, human-exoskeleton robot interfaces, and so on. None, however, have focused on the coordination of control between humans and these kinds of robots. In this paper, we examine the research regarding human-exoskeleton robot coordinated control to capture the current state-of-the-art. One hundred and fifty six relevant articles were collected and screened from the Web of science, and classified into six categories, based on human neurobiology and exoskeleton robots' assistive effects. These categories were further subdivided according to their perspective on human-exoskeleton robots coordinated control. The paper extensively reviews various control methods we uncovered and how they are coordinated between wearer and exoskeleton robot.
\end{abstract}

\section{Key Words}

Lower limb exoskeleton robot, human-robot coordination control strategy

\section{Introduction}

There have been studies regarding the potential of lowerlimb exoskeleton robots for more than fifty years now and over the past decade significant public interest in the domain has started to develop. There has also been a massive surge in the variety of lower limb exoskeleton robots being made by different institutes all over the world, with

* Guangdong Provincial Key Laboratory of Robotics and Intelligent System, Shenzhen Institutes of Advanced Technology, Chinese Academy of Sciences, Shenzhen 518055, China; e-mail: \{yue.ma, can.wang, cj.chen\}@siat.ac.cn

** Shenzhen College of Advanced Technology, University of Chinese Academy of Sciences, Shenzhen 518055, China

*** Department of Mechanical and Automation Engineering, The Chinese University of Hong Kong, Hong Kong, China; e-mail: xy.wu@siat.ac.cn

**** Department of Mechanical and Aerospace Engineering Rutgers, The State University of New Jersey, New Jersey, USA; e-mail: jgyi@rutgers.edu Corresponding authors: Xinyu Wu

Recommended by Prof. Anmin Zhu

(DOI: 10.2316/J.2019.206-0193) different types of mechanical structure and actuators and with different control strategies. Exoskeleton robots have exhibited the potential to enhance an operator's range of possible movements across numerous military, medical and industrial applications. Depending on the application and their intended functionality, lower-limb assistive robots can be roughly classified into two categories: power assistive and rehabilitation. Some kinds of power assistive exoskeletons can be used for carrying heavy loads. Here, the loads are put in a backpack that is part of the exoskeleton and the wearers then guide the exoskeleton's movements. Examples here include BLEEX [1]-[3], HULC [4],[5] and Hercule [6]. Faced with the urgent needs of an ageing population, other such robots have been designed to enhance the walking ability of old people and their capacity to ascend and descend stairs (see, for instance, HAL [7] and Honda's Stride Management Assist [113]). When it comes to rehabilitation, some exoskeleton robots are used to replace the repetitive rehabilitation activities required of therapists and to increase patient participation in rehabilitation training (see, here, systems such as LOPES [138], [130] and ALEX [28]-[30], [114], [141]-[143]). Others have been used to help paraplegic or quadriplegic patients regain their ability to walk in ordinary everyday life, e.g., Ekso [62], Indego [61] and ReWalk ${ }^{\mathrm{TM}}$ [59]. In summary, lower limb exoskeleton robots have played a significant role in assisting people's mobility and look set to play an equally important role in the future.

A number of review papers have been published that provide a good overview of the key technologies associated with exoskeleton robots. Tingfang et al., for instance, summarized 76 articles between 1990 and 2014 according to different assistive control strategies [8]. Six different types of control methods were identified. However, human-exoskeleton interaction methods related to these control structures were not discussed. This includes EEG-based control strategies and multi-model fusion control strategies. This may have been because, at the time, relatively few papers talked about such matters. Domen and Robert [9] presented a review of sensor fusion methods for wearable robotics in 2015 that provided lots of detail about sensor fusion-based approaches, including the use of EEGs and EMGs. However, they did not relate these to 
control structures. Wei et al. [10] set about summarizing the mechanical structure, training mode and rehabilitation control strategies of lower-limb rehabilitation exoskeleton robots published between 2001 and 2014. They focused, in particular, on the clinical effects of different training modes and control rehabilitation algorithms over that period. Unfortunately, they only reviewed rehabilitationoriented exoskeletons. The techniques associated with power assistive exoskeletons were not discussed. Michael et al. [11] reviewed the key technologies used for controlling lower-limb prosthetic and exoskeleton devices. Concentrating on how these devices interacted with the human sensory-motor control system, they moved on to propose a general hierarchical gait assistance control framework. Allan and Quan [12] systematically reviewed the actuators used in exoskeleton robots, including electric, hydraulic and pneumatic varieties, and analysed the extent to which they met their application requirements. This work was used to critically evaluate current actuator technologies and examined potential future exoskeleton robot actuator technologies. Michael et al. [13] identified a total of 21 different active lower limb exoskeleton robots that aimed at restoring impaired functional performance and discussed variable impedance actuation units, control systems that could react to wearer intent and mechanical designs that could deliver high force/torque.

Although the above review papers have summarized research regarding mechanical design, actuators, control systems and the use of sensors in exoskeleton robots, a systematic review of how to bring about human-exoskeleton coordination and coordinated control strategies is still lacking.

Human-exoskeleton coordination is important because these kinds of robots are strongly coupled human-machine interaction systems where each party's movements can have a major impact on the other party. In this paper, we will be examining how exoskeletons can coordinate with humans in concert, especially from the point of view of human neurobiology and motor circuits. By summarizing exoskeleton coordination control strategies from this perspective, we are able to observe existing limitations and illuminate potential future directions for development.

The rest of this paper is organized as follows: In Section 2, we are going to introduce the literature search methodology used for the paper and delineate the scope of the review and the typologies of coordination control strategies we shall be drawing upon. In Section 3, we will be reviewing the coordination strategies in terms of the input and output of human motor circuits. Finally, in Section 4, we will discuss current research limitations and future directions and provide our conclusions.

\section{Literature Search Methodology and Classification Approach}

In this section, we will introduce the search methodology we adopted. Then we will provide a thorough description of how we approached the classification of our materials. After this, we will summarize various coordination control strategies and associated articles to give an overview of the paper.

\subsection{Search Methodology and Scope}

To assemble a collection of papers in line with the goals of our review, we searched the Web of Science using a set of keywords. The keywords were as follows: Topic $=\left({ }^{*}\right.$ lower leg* OR *lower-limb* OR *lower-extremity* OR leg OR hip OR foot OR knee OR ankle) AND Topic $=\left(\right.$ ortho $^{*}$ OR exoskeleton* OR wearable robot* OR portable robot* OR robot suit) AND Topic $=$ (power* OR active OR artificial $^{*}$ OR intelligent* $)$ AND Topic $=($ aid OR assist* OR improv* OR augment* OR enhance* OR reinforc*) NOT Topic $=\left(\right.$ prosth $^{*}$ OR $*$ artificial limb*). With the above keywords, we initially retrieved a total of 3,096 publications from across the selected database (the publications were dated up to December 2017). The research field was set to "science and technology" and the document types were set to "articles and meetings". After filtering, 2,499 publications remained. We now set the research areas to engineering; computer science; robotics; and automation control systems. This left us with 917 papers, out of which 7 were repeated. Topics related to exoskeleton robots can include gait evaluation researches, medical skeleton image researches and powered prosthetic system research, so these were excluded manually. We were now left with 416 papers. In this paper, we wanted to review human and exoskeleton robot coordinated control. We therefore excluded topics regarding mechanical design, sensors and the electronic design of exoskeletons. The remaining 158 papers were kept to review in more detail. The rest of this article draws upon these 158 papers for its discussion and analysis of technologies related to human-exoskeleton robot coordination control strategies.

\subsection{Classification}

To research coordination control methods, human motion control strategies need to first be understood. Humans have three types of motion: reflex, rhythmic motor pattern and voluntary movement. Reflex does not require intention from the brain. It is motion automatically stimulated by a particular sensation. A rhythmic motor pattern also does not need control from the brain. It self-regulates to perform rhythmic movements such as gait and arm swings during walking. The combination of rhythmic signals is generated by the central pattern generator (CPG) in the spine. Voluntary movement requires the participation of the brain and can complete all sorts of complex movements. Depending on the type of motion, there are four sources from which human motion intention might be obtained: the brain; the spine; muscle fibre; and limb movement. Signals from the brain, spine and muscle fibre are physiological signals. Signals related to limb movements, such as position, velocity and muscle contraction, are physical signals.

Inputs to the exoskeleton coordination controller are the human physiological or physical signals described above, or a combination of the two. However, the technology is only able to obtain human intention from brain activity and muscle fibre myoelectricity at present. There are generally three kinds of assistive output from the exoskeleton coordination controller: position (joint angle), 


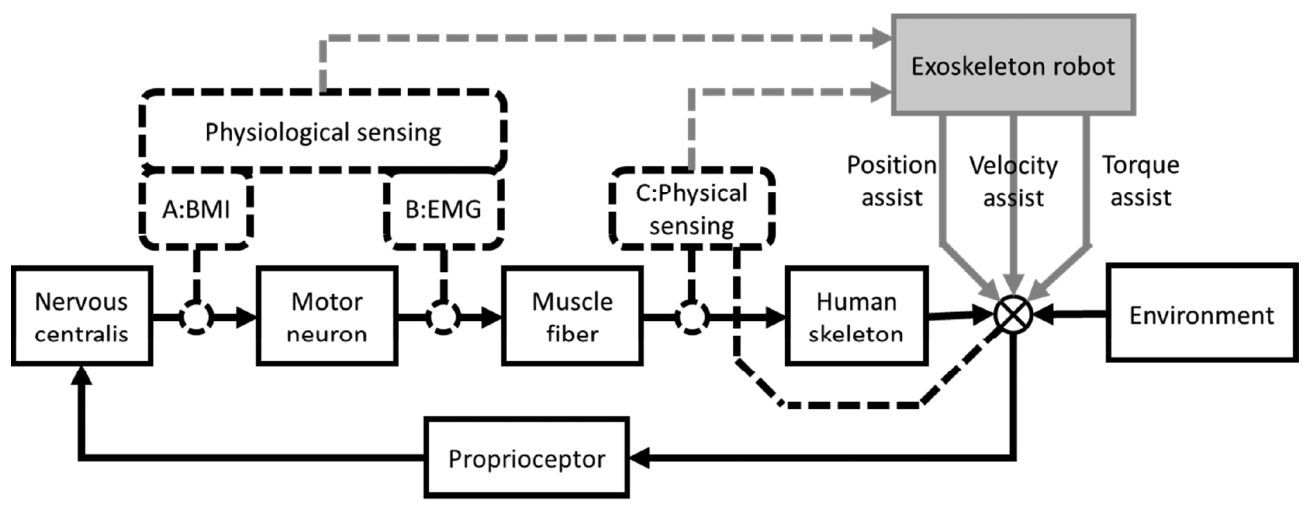

Figure 1. Control structure for human-exoskeleton robot coordination. The coordination controller for the exoskeleton robot obtains human intentions via physiological and physical sensing. At the same time, it generates assistive torque, velocity or position to complement the human body. The feedback loop between them becomes the human proprioceptor. A is the brain machine interface (BMI), which acquires motion intention from the nervous centralis; B is the myoelectricity obtained from the muscle fibre; $\mathrm{C}$ is the physical sensing that captures things like muscle contractions, heel strikes, position, velocity, and so on.

Table 1

Classification

\begin{tabular}{|l|l|l|c|}
\hline Inputs & Outputs & Control strategy & Number of articles \\
\hline \multirow{4}{*}{ Physical signal } & \multirow{2}{*}{ Position } & Gait trajectory based position assistance & 45 \\
\cline { 3 - 4 } & & Posture estimation based position assistance & 7 \\
\cline { 3 - 4 } & Velocity & Interaction force based velocity assistance & 5 \\
\cline { 3 - 4 } & \multirow{2}{*}{ Torque } & Posture estimation based torque assistance & 37 \\
\cline { 3 - 4 } & & Interaction force based torque assistance & 26 \\
\cline { 3 - 4 } & & Gait feature estimation based torque assistance & 12 \\
\hline Physiological signal & Position & Physiological intention estimation based position assistance & 7 \\
\cline { 2 - 4 } & Velocity & Physiological intention estimation based velocity assistance & 1 \\
\cline { 2 - 4 } & Torque & Physiological intention estimation based torque assistance & 18 \\
\hline
\end{tabular}

velocity (joint angular velocity) and torque. The exoskeleton coordination control structure is summarized in Fig. 1 with a human in the loop. Human motion intention can be detected from sources $\mathrm{A}, \mathrm{B}$ and $\mathrm{C}$. A and B are the physiological signal inputs, $\mathrm{C}$ is the physical signal input.

In the next section, the content is classified according to the inputs and outputs of the exoskeleton coordination controller. Some of the research that combines physiological and physical signal input is classified as physiological. The resulting classification is summarized in Table 1. Several coordination control strategies are included for each input and output (nine categories in total). The following sections will discuss the various coordination control strategies in more detail.

\section{Results}

In this section, we will be discussing the nine categories of coordination control strategies. For each category, we will first present the main idea and control structure, then we will discuss different technical details of different exoskeleton robots. We will conclude each discussion with a short commentary.

\subsection{Gait Trajectory-based Position Assistance}

This coordination strategy establishes a coordinated movement relationship between a human and an exoskeleton robot through gait. One body of research here focuses on using or developing a gait that is as closely suited to the human operator (or pilot) as possible. However, as a part of this, the pilot needs to learn how to cooperate with the exoskeleton robot and to understand what the robot is actually capable of. There are three main aspects to this control strategy in that case: how to generate a suitable gait (or adapt it accordingly); how to design the position controller itself; and how to ensure that the pilot and the gait component of the exoskeleton are properly coordinated. This coordination control strategy is summarized in Fig. 2. The pilot is physically coupled with the mechanical 


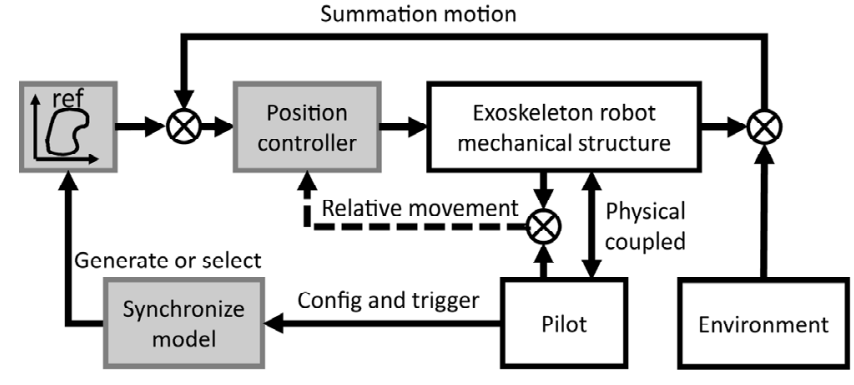

Figure 2. Control structure for gait trajectory-based position assistance.

structure of the exoskeleton robot and they move together. The relative movement of the lower limb and the exoskeleton is deemed a disturbance or a compliance parameter of the position controller in this strategy. The pilot can configure the gait and trigger the exoskeleton robot's synchronization model if necessary. The gait trajectory is generated or selected and entered into the position controller. A closed position control loop resolves any environmental and piloting effects and tracks the reference trajectory.

\subsubsection{The Position Controller}

Generally, a simple proportional-derivative (PD) controller is employed as the position controller in gait orthosis [32], pneumatically operated gait orthosis (POGO) [52], Mina [20], [54], Lokomat [58] and CUHK-EXO [33]. The advantage of a PD controller is that it can reach a target angle within the degrees of compliance necessary to ensure safety. At the same time, there are a few articles that mention using a proportional-integral-derivative (PID) controller [55], [34] and a proportional controller [36] to track preset trajectories.

For safety reasons, the analysis and development of compliance controllers have become a particular focus of research. ATLAS [53]-[55] employs an active compliance controller that measures the force registered by the insoles. ALEX [14], [15] and C-ALEX [16] employ a force field controller (FFC) to facilitate neuromuscular plasticity and improve walking ability. The FFC creates tunnel-like virtual walls for an ankle point that is following an ankle point trajectory using Cartesian coordinates. Within the virtual wall, the controller offers a degree of compliance. C-ALEX is a cable driven exoskeleton that has a low-level cable tension controller to implement the FFC control. Heo et al. [17] have employed an impedance controller that imposes a safety margin on the tracking errors generated by a bicycle-riding assistive exoskeleton robot. The impedance controller modifies the impedance properties according to the measured interaction forces. This method is similar to ALEX. The KNEXO [31]-[33] innovatively employs Pleated Pneumatic Artificial Muscles (PPAMs). These are actuators for powered exoskeletons that are designed to be inherently compliant. In [18], the controller makes the actuators track reference gait trajectories, allowing for large trajectory deviations whilst still ensuring safe guidance. The controller is based on a Proxy-Based
Sliding Control (PSMC) method. Ahmed et al. [21], [22] have employed a recursive least square estimator (RLSE) for their variable admittance controller (VAC), which can adjust the position controller's stiffness and damping values. Hu et al. [23] have employed an impedance control for their exoskeleton robotic iLeg intended for rehabilitation applications. The controller has a dual closed loop structure. The outer loop is an admittance control loop that is used to regulate the interaction force between the patient and the robot by modifying the reference trajectory. The inner loop uses a PD control algorithm where compensation is handled by back propagation neural networks (BP $\mathrm{NNs}$ ). The impedance parameters are determined by fuzzy logic to provide an adaptive haptic interface. The ELLTIO [24] exoskeleton employs a PD control law with adaptive compensation to follow a desired rehabilitation routine's trajectory. The adaptive control law commonly used in continuous adaptive systems is often called the integral law or gradient type. Ugurlu et al. [25] have proposed a VAC that can adjust the reference trajectory adaptively in a way that complies with force constraints. The VAC processes both position and force constraints simultaneously and the controller manages the trade-off between position and force automatically via virtual admittance coefficients. Jamwal et al. [43] have employed a boundary layer augmented sliding mode controller (BASMC) to control a pneumatic muscle actuated (PMA) parallel ankle rehabilitation robot. PMAs not only are intrinsically compliant but also have some variabilities that can still be managed through the dynamic model. The controller not only offers robust trajectory tracking performance but also enables deviations from the reference trajectories. In [27], an ankle rehabilitation exoskeleton uses a similar position control structure, while the controller replaces the BASMC with an adaptation law. Rifai et al. [28], [29] apply a different control method in their EICOSI exoskeleton robot. In [28], they put forward an $L 1$ augmented adaptive controller for position control. This control algorithm can decouple robustness and adaptability. State errors are measured between various, whilst a projection method ensures the boundedness of the system. In [29], the EICOSI uses a bounded control strategy to avoid saturation of the actuators when tracking a desired trajectory. The lowest self-defined threshold saturations for joint angles and joint velocity errors are used to avoid actual saturation. Santos et al. [30] have designed a rotary series elastic actuator (RSEA)-driven knee orthosis system. A cascaded controller is employed with a position loop cascade containing a torque loop and a torque loop cascade containing a velocity loop. The position loop uses a PD controller. The torque loop is based on $k_{\infty}$ criteria to ensure good system performance when faced with parametric uncertainties and external disturbances. The velocity loop uses a PI controller. Li et al. [31] have proposed a multi-mode control scheme for rehabilitation robotic exoskeletons. The scheme is composed of three control modes defined by different error regions. Each mode can be switched to smoothly. Using this approach to control rehabilitation, exoskeletons are able to "assist-as-needed", whilst guaranteeing pilot safety. 


\subsubsection{Gait Trajectories}

Gait trajectory can be defined in multiple ways. The DGO [32] uses a predefined gait pattern and a treadmill speed that can be adjusted by a therapist. Included here is a user interface where a therapist can adjust the gait pattern easily. The EICOSI [28], [29] exoskeleton robot employs a therapeutic doctor defined gait trajectory. POGO [52], Mina [53], [54], KNEXO [19] and CUHK-EXO [33] all have a zero torque mode that obtains reference trajectories from normal people. Drawing upon the tracked gait of normal people's natural walking patterns, the zero torque mode avoids applying unnatural forces/torque to the pilot. This is a classic teach and replay mode for fixed reference trajectory assistance that is employed by many systems, e.g., [34]. AUSTIN [25] and an exoskeleton designed by Banchadit et al. [36] make use of Winter's clinical gait analysis (CGA) [37] data as its optimal reference gait trajectory. WPAL [38], meanwhile, uses a fixed parameter-determined reference trajectory to assist paraplegic patients.

Some gait trajectories are generated by mathematical models. These trajectories can change certain characteristics according to the information derived from sensors. In this assistive approach, the wearer takes the leading role in the coordination, giving the wearer a sense of control. Low et al.'s [39], [40] NTU wearable exoskeleton system combines an inner exoskeleton with an outer exoskeleton. The inner exoskeleton is equipped with encoders that obtain the motion of the pilot without employing any actuators that might cause obstruction. The outer exoskeleton is then capable of carrying heavy loads. The gait trajectory of the outer exoskeleton is taken from the inner exoskeleton, with the human operator controlling the inner exoskeleton's leg intuitively. A zero moment point (ZMP) algorithm is used to keep this system in balance. Kim et al. [41], [42] have proposed a gait trajectory planning method that uses function distribution analysis and dynamic manipulability ellipsoid (DME) theory. This method quantifies the walking characteristics and dynamic performance of an exoskeleton and uses DME to capture the relationship between the angular velocity and the line velocity of the robot's links. Sanz-Merodio et al.'s [53]-[55] ATLAS robot was developed to enable quadriplegic children to walk. ATLAS employs a parameter-based method to generate reference joint trajectories. All of the gait feature parameters in every step can be changed. The supporting leg employs a parameterized linear inverted pendulum method, in which the height and the length of a stride can be configured. The swinging leg employs a self-defined function related to the step length and height. Trung et al. [46] have developed a two DOF-based orthosis system to use with hemiplegic patients who have one healthy leg and one disabled leg. Here, two control strategies run in parallel. One system acquires position control signals from the healthy leg that are then transmitted to the disabled leg after a fixed delay. As a patient's speed changes, the delay time changes as well. The other control system runs an algorithm that draws exclusively upon the angular information to generate an approximate trajectory with sinusoidal signals. This algorithm also has a modifiable delay time. Hasegawa et al. [47], [48] have developed a finger-mounted human-exoskeleton interface with a human-exoskeleton cooperative controller that can help fully paraplegic patients to walk again. Three finger joints are mapped to the joints of the exoskeleton to generate the gait using potentiometers. The controller divides the gait into six phases. Phases 1-3 describe the motion of the crutch and make the exoskeleton balance. Phases 4-6 map the finger movements to the joint angles of the exoskeleton. In [48], they describe a finger-mounted walking controller that can be configured using self-designed force sensors and an electrode array. The force sensors are used for controlling the extension/flexion angle of the lower limb exoskeleton. In an innovative move, they use the electrode array to feedback the foot position of the exoskeleton robot. The pilot controls the step length in the swing phase using the force sensor and the exoskeleton controls the weight support in the support phase. Ugurlu et. al. [25] generate motion by means of a predefined centre of mass (CoM) position in the $x$-axis, the pitch orientation of the upper body and the foot trajectory in the $y$-axis and $z$-axis, respectively. This is similar to a ZMP theory-based motion generation method. A smooth and continuous trajectory is synthesized via polynomial interpolation. Kagawa et al. [49] have developed a smooth motion planning approach for assistive walking exoskeleton that is based on a minimum jerk trajectory algorithm. Here, a linear inverted pendulum model is used to prevent falling down during walking and a continuous trajectory is planned by the minimum jerk trajectory algorithm. Four points are defined in a task space by the pendulum model that determine the shape of the continuous trajectory: the initial point; via-point 1 ; via-point 2 ; and the final point. Via-point 1 is a parameter-determined point based on the linear inverted pendulum model. The final point is determined by the stride length and the orbital energy at the end of each step. Via-point 2 provides an optimized state. These four points are converted to joint motion by the minimum jerk trajectory algorithm. Mina v2 [50], which came second in the 2016 Cybathlon Powered Exoskeleton competition, is designed to help paraplegic patients regain walking ability. The Mina v2 has six active DOFs in the sagittal plane in the hips, knees and ankles. These characteristics, derived from the mechanical structure of active ankles, are used to add a weight transfer phase that offers a large degree of toe-off. To make the planned gait adaptable to different terrains, they proposed a novel method for finding the joint position based on the swinging foot position in Cartesian space. A minimum jerk motion planning method is then employed to ensure continuous trajectory in joint space. The Mina v2 has four kinds of leg trajectory: swinging leg trajectory, transfer trajectory, stairs trajectory and ramp trajectory. The gait planning parameters can also be adjusted Online. Kamali et al. [51] have proposed a trajectory generator based on dynamic movement primitives (DMPs) theory to assist the sit-to-stand (STS) motion of the FUM-KneeExo. The DMPs model is trained by a library of sample trajectories and the initial joint angles are input to this model to predict the desired STS 
motion of the pilot. A self-defined trajectory function is used as the training trajectory. The DMPs model provides a time-normalized and flexible trajectory planning framework. Relatedly, Ahmed et al. [21], [22] have developed a control algorithm that applies adaptive central pattern generators (ACPGs) to generate gait trajectories.

\subsubsection{The Synchronized Model}

To make the gait adaptable to the pilot, researchers have adopted a number of different approaches. Experiments using POGO [52] have shown that pilots find it hard to synchronize with exoskeleton robots when using fixed reference trajectory assistance, even if the walking gait is obtained from their own. As a result, a feedback synchronization algorithm was designed that compensates during the desired walking phase by assessing the error between the desired and the actual stepping period. Similarly, Ahmed et al. [21], [22] have employed ground reaction force sensors to adjust the walking speed. The Mina [53], [54] uses a musical instrument digital interface (MIDI) to adjust the recorded walking speed in a range of 50\%-130\%. In the exoskeleton robot designed by Lugris et al. [55], the shape of a normal person's reference trajectory is scaled in time so that it can be adapted to a desired gait speed, like the Mina. Yang et al. [56] employed an adaptive gait trajectory control strategy to eliminate asynchronization during the gait training of the LRER exoskeleton robot. They constructed an inverse dynamic model to predict active torque which is bringing about by patients, then used a fuzzy adaptation algorithm to accommodate different walking patterns. At the same time, the controller limited the boundary of the gait to avoid awkwardly posed trajectories. Zhang et al. [57] also used a gait trajectory adaption strategy to control a four DOF lower limb rehabilitation exoskeleton. The main idea, here, was to modify the predefined profile of the exoskeleton according to the joint reaction torques between the exoskeleton and the pilot using a self-constructed human-exoskeleton interaction model. Lokomat [58] is a well-known commercialized rehabilitation device. Lokomat controls the walking trajectory of its exoskeleton robot and the speed of its treadmill simultaneously. Reference walking trajectories are converted into a time-independent class. A window moves at a predefined speed along the walking trajectory and the scope of the trajectory is limited to the moving window. The speed of the treadmill is controlled by an Automatic Treadmill Speed Adaptation (ATSA) approach that measures the interaction forces. The exoskeleton and the treadmill are synchronized via a gait phase strategy.

\subsection{Posture Estimation-based Position Assistance}

This coordination strategy assists the pilot by obtaining the posture of the coupled human-exoskeleton system. In the position assist mode, the pilot's limbs are not free to move, but the pilot can express their motion intent through arm movements or an unconstrained part of the current joint and foot set-up, thus using body synergy to determine the kind of position assistance that is provided. There are

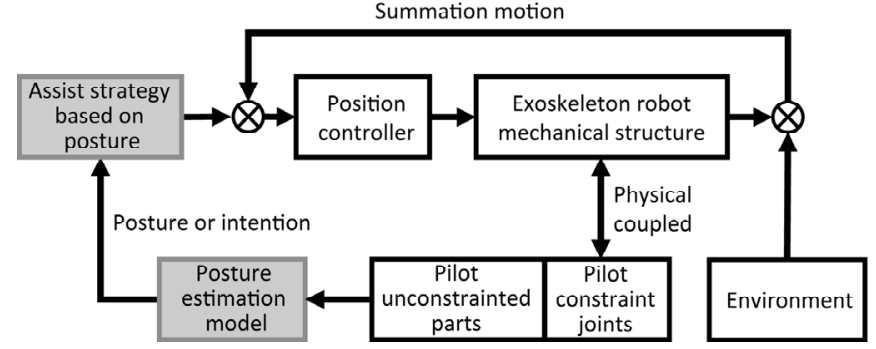

Figure 3. Control structure for posture estimation-based position assistance strategies.

two main aspects of this assistance strategy: obtaining the pilot's intention from their posture; designing an assistance strategy based on this posture. The control structure of this coordination strategy is summarized in Fig. 3. The posture estimation model and various assistance strategies based on it are discussed below.

\subsubsection{Posture Estimation Model}

There are many ways of acquiring a pilot's intention based on their posture. In the ReWalk ${ }^{\mathrm{TM}}$ [59] exoskeleton, a tilt sensor is placed on the pelvis of the robot that automatically triggers bipedal walking. Strausser et al. [60] use foot pressure sensors across the entire foot of a medical exoskeleton robot based on HULC to determine the posture of the pilot when helping paraplegics to walk. This is the most commonly used method for controlling lower limb exoskeleton robots. Poonsiri et al. [65] use a similar method to determine the motion, whilst Quintero et al. [61] control Vanderbilt-powered orthosis by estimating the location of the centre of pressure $(\mathrm{CoP})$, which is once again sensed by posture sensors. When the CoP moves to the forward foot within a predefined distance, a step is triggered. The eLEGS [62] exoskeleton has angle sensors embedded in its hip and knee joints, foot pressure sensors, angle sensors on its upper arm and crutch load sensors that are used together to determine body posture. The arm angle is specifically used to get the walking intention of the pilot. A threshold is set by observing the angle of the user's arm when they want to walk and put forward their crutch. To ensure pilot safety, the crutch load, foot pressure and lower limb angle are used to make sure that the pilot is ready to move forward. Both ATLAS [63] and Kinesis [64] use ground reaction force (GRF) sensors in the heel and angle sensors in the knee and hip to drive a state machine-based controller.

\subsubsection{Assistance Strategy Based on Posture}

In the ReWalk ${ }^{\mathrm{TM}}$ [59], a tilt sensor determines the trunk angle and triggers a prescribed hip and knee displacement (angle and time) that results in a step. The medical exoskeleton robot based on HULC to help paraplegics walk designed by Strausser et al. [60] uses a finite state machine (FSM) model to assist the pilot. Walking, here, is divided into four states: stance, toe-off or latent stance, swing and heel strike. The state transitions are determined by foot pressure sensors. The trajectory during the swing is 
generated using CGA data. A PD controller is employed during the swing to track the trajectory and ensure its compliance. During the stance posture, the knee is held straight. The Vanderbilt-powered orthosis [61] also uses a FSM to assist the pilot. The FSM consists of 12 states. The transition between each state is triggered by the position of the CoP. The Vanderbilt-powered orthosis, here, was subsequently converted into a commercial product called Indego. The eLEGS [62] software uses a FSM based on walking states too, controlling the exoskeleton to assist patients with walking disorders. The walking cycle is divided into four simple, separate states: left swing; left double stance; right swing; and right double stance. The transitions between each of these states are managed by sensors, as discussed in Section 3.2.1. ATLAS [63] once again uses a FSM for control purposes. The hip and knee joints have two separate FSMs to adjust the gain of the PD controller. In each state, the gain is different so as to allow free movement and pursuit of a desired trajectory. The transitions between each state are determined by foot pressure and the angle of the joints. Kinesis [64] is a hybrid actuated exoskeleton robot with functional electrical stimulation (FES) that is used for walking rehabilitation. Here, a FSM is employed to coordinate the FES and joint controllers. The joint controllers track a reference trajectory that allows for deviation. The FSM operates in a cycle domain (c-FSM) and a time domain (t-FSM). The t-FSM broadcasts gait cycle events and coordinates the c-FSM for the left and the right leg. Poonsiri et al. [65] prefer a simple control strategy. When the posture is in a double stance state, the exoskeleton extends and when the posture is in a single stance state, the knee joints bend to a maximum of $50^{\circ}$. Once the foot is lifted, the links in the exoskeleton straighten again to prepare for the next cycle. The position of the joint angle is controlled by a PID controller.

\subsection{Interaction Force-based Velocity Assistance}

In this coordination strategy, wearers are provided with velocity assistance by measuring the interaction force between the wearer and the exoskeleton robot. The control structure is summarized in Fig. 4. Interaction force is the chief input for this control system. When pilots move their limbs and the exoskeleton blocks the movement, an interaction force is generated. This is converted into velocity to control the exoskeleton's movements. Ideally, a velocity controller will make sure that the system can track the input as accurately as possible.

Chen et al. [66] have proposed a force-velocity control algorithm to control the PAWL exoskeleton robot. The force-velocity control algorithm is based on a humanexoskeleton dynamic coupling model. The inertia of the pilot and the exoskeleton are precisely measured to control the exoskeleton, though the velocity controller itself is not discussed. Kong and Jeon [67] have employed an interaction force-based velocity and torque assistance strategy with the tendon-driven exoskeleton EXPOS, which is designed to assist elderly people. The interaction force is measured by an air bladder and a pressure sensor. Using

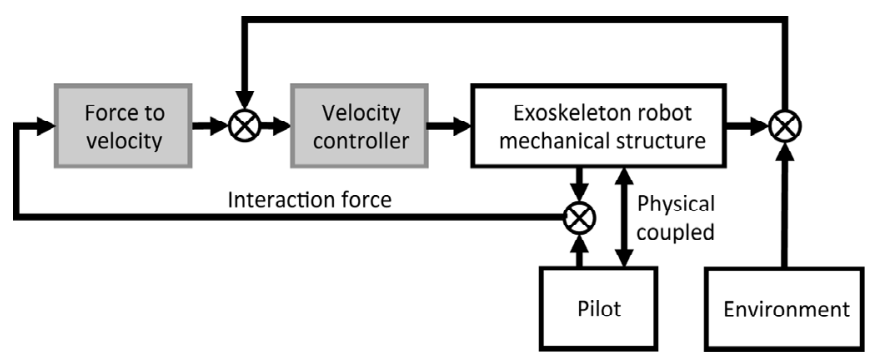

Figure 4. Control structure for interaction force-based velocity assistance strategies.

these methods alone, it is difficult to obtain the interaction force in free mode (e.g., if there is no motion but the muscle is contracted or relaxed). A fuzzy logic-based controller is therefore also used to overcome this problem. In the free mode, the motor control depends on joint velocity and the exoskeleton provides torque and velocity assistance across different states. Weinberg et al. [68] have designed an Active Knee Rehabilitation Orthotic Device (AKROD) that also employs velocity assistance in its isokinetic profile mode to correct abnormal leg gait and excessive stance-related knee extension for stroke survivors (which is important from a rehabilitation point of view). However, interaction force is not used to control the velocity of the exoskeleton when being used for rehabilitation. Instead, a simple PID controller is used for the velocity. Wu et al. [69] have adopted two control methods for the powered hip exoskeleton PH-EXOS to assist pilots with walking impediments. The first is a cascaded PID controller for reference trajectory tracking. The second is a fuzzy adaptive controller to provide walking assistance. A fuzzy adaptive law is used to adjust the parameters and calculate the velocity of the hip joints. Zhang et al. [70] used a similar strategy to design the load-carrying enhancement robot HIT-LEX. Here, interaction force is obtained from a set of self-designed sensors that measure the interaction force in the feet and the back. An expected terminal velocity is obtained using the current speed and expected speed increments. The control scheme primarily focuses on terminal velocity estimation and calculates the expected velocity for each driving joint.

\subsection{Posture Estimation-based Torque Assistance}

This coordination strategy is similar to posture estimationbased position assistance, but does not constrain the motion of the pilot. A feeling of resistance only arises if the robot and the pilot are not in synergy. Posture estimationbased position assistance has to solve two problems: how to get the posture information and intention of the pilot from the posture? How to design an assistance strategy based on the posture? The control structure is summarized in Fig. 5. The posture estimation model and the assistance strategy based on the posture are discussed below. To distinguish this from the gait feature-based torque assistance discussed in Section 3.6, we consider continuous gait phase estimation, cadence and walking speed as gait features. 
Summation motion

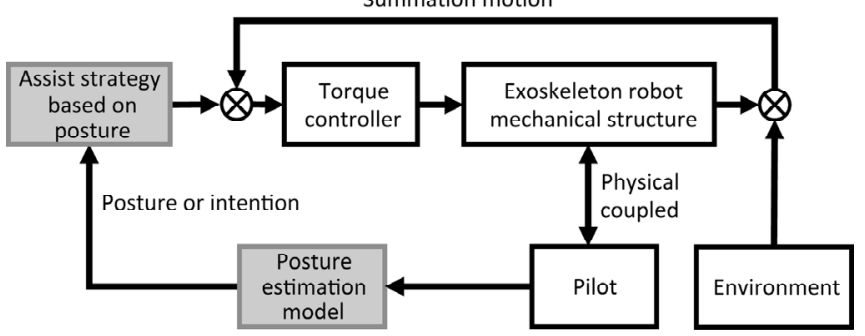

Figure 5. The control structure of posture estimation based torque assistance strategy.

\subsubsection{Posture Estimation Model}

Fujishiro et al. [71] use a threshold method based on a load cell sensor under the foot to determine two postures: whether the foot is on the ground or not. Chen et al. [72] also employ a threshold method, this time based on angles, angular velocity and floor reaction force to estimate posture. Low et al. [73] have designed a rotary encoder that uses two photo detectors to identify the direction and the magnitude of knee joint movements. Walsh et al. [78]-[80] have adopted a SEA as the actuator. The knee angle and interaction force are measured with a threshold method being employed for switching between states. Oymagil et al. [77] use a FSR and an angular potentiometer to obtain heel strikes and leg swing information, respectively. Adopting a threshold approach, these signals are used as a switch. Sugisaka et al. [78] employ two types of pressure sensor to get the state of the pilot and adopt a threshold method for switching between states as well. Sulzer et al. [79] use treadmill load cells to measure step on and step off events. Kim et al. [80] use four FSR sensors as ON-OFF switches to indicate ground contact. Morris et al. [81], meanwhile, use two different methods to obtain gait events. One is similar to [78], where two pressure sensors are put on the heel and toe to estimate the walking state, with the torque being assisted accordingly. The other method estimates gait events using a cross-correlation (CC) algorithm based on signals from accelerometers, potentiometers and force sensors. This method establishes a relationship between the sensory signals and gait cycle through regression analysis. Sasaki et al. [82], [83] also use FSR as a threshold-based contact switch to get step events. In an approach similar to switch mapping, Shamaei et al. [84] determine gait events according to the direction of the knee joint velocity and the GRF state of heel. Shen et al. [85] determine posture using kinematics and the GRF state in a way similar to [84]. The switch thresholds are determined through off-line analysis. In [86], a Gaussian Mixture Model (GMM) is proposed that uses a ground walking gait event detector to estimate posture and the walking state. This detector can estimate six major periods in a gait cycle. Wehner et al. [87] sense heel strikes via footswitch-instrumented insoles. The foot on/off states are used in a soft exosuit. Mooney et al. [88] use the angular velocity of the shank in the sagittal plane to distinguish stance events and swing events.
Somewhat differently, Kanno et al. [89] take advantage of the back-drivability of pneumatic actuators to detect pilot motion. To get an effective base pressure, EMG sensors are also used for pressure calibration. Kim et al. [90], [91] use four one-axis load cells under the foot to detect gait events. The sum of the load cells is classified into stance and swing phases, again by means of a threshold. Lim et al. [92] use a similar method, whilst Ka et al. [93] have designed an under-foot air pressure sensor system to detect CoG movements in the coupling system during walking and detect the swing and stance events between the two legs. Zhou et al. [95] use plantar pressure and knee joint velocity to classify five gait events according to a threshold, including early stance; middle stance; late stance; swing knee flexion; and swing knee extension. Hyun et al. [96] have designed a flexible rubber shoe insole embedded with two force sensing resistor (FSR) sensors. This was installed inside a commercial shoe to estimate a wearer's gait phase including the air, heel-strike, support (full contact) and toe-off. Lerner et al. [97] also use FSR sensors to distinguish transitions between stance states and swing states. The angle and the angular velocity of the knee joints were used to measure transitions between what the Rancho Los Amigos (RLA) terminology calls early and late swing phases. Thresholds are calculated based on offline data. In an approach similar to [95], the thresholds are adjusted when used Online during the first walking session. Thapa et al. [98] measure the GRF using plantar pressure sensors to provide an estimate for assistive torque in the loading phase. The joint angle and the angular velocity of the knee joints were measured to estimate the necessary assistive torque in the rising phase.

\subsubsection{Assistive Strategies Based on Posture}

FSM-based methods are the classic approach to assistive strategies. Fujishiro et al. [71] employed an ON/OFF strategy to control a pneumatic assistive system to help handicapped or injured people when walking. As a step is detected, the system assists knee joint extension by providing the correct air pressure to a rod-less cylinder attached to the side of the knee joint. Chen et al. [72] have designed a leg exoskeleton robot that innovatively employs an MR actuator at the knee joint. Here, stance extension and stance flexion of the knee joint are assisted by the actuator but no assistance is provided in the swing phase. The assistive torque is proportional to the estimated joint torque. Low et al. [73] provide assistive torque according to the direction and encoder pulse of knee movements delivered by a self-designed sensor. The motor maintains torque with every pulse from the encoder. Walsh et al. [78]-[80] have designed a quasi-passive leg exoskeleton for augmenting a pilot's strength when carrying heavy loads. They implemented two different actuation strategies at the hip joint. The first strategy placed uni-directional springs at the hip joints. The second strategy placed a SEA actuator at the hip joints instead. In the second strategy, the control method imitated the springs to work out the joint torque. The hip motion was divided into five states during the walking cycle. The knee motion was divided 
into four states. During the different states, the different torques were rendered proportional to the angular deviation. Oymagil et al. [77] have designed a SEA-driven ankle joint exoskeleton for stroke rehabilitation using a treadmill. The gait pattern in the model is divided into four different states: heel strike; stance; swing; and late swing. Each state is a linear vector representing the position of the motor that can be translated into torque by the SEA actuator. Sugisaka et al. [78] have developed an artificial muscle-driven ankle-foot orthosis. Their design incorporates a four-state FSM controller: heel touches down; toe touches down; heel leaves; and toe leaves. Different pressure change functions are applied to the different states. Sulzer et al. [79] have designed a series elastic remote knee actuated (SERKA) exoskeleton that can selectively assist knee joints in stroke patients with a knee disability. The assistive strategy has two states: a stance foot event; and a swing foot event. A self-defined torque function for different states is applied, with the proportion of assistance being adjusted manually using a visual check of the knee flexion angle. Kim et al.'s [80] SEA-driven active ankle joint exoskeleton aims to prevent foot drop and toe drag when hemiplegics are walking. A four-state FSM controller is employed covering: HS (heel strike); FF (foot flat); HO (heel off); and TO (toe off). After HS, the controller shortens the actuator to induce plantar flexion, so that the foot is placed flat on the ground to support the body's weight. During FF, the actuator is lengthened to induce dorsiflexion. HO occurs after FF, during which the controller shortens the actuator rapidly to induce plantar flexion to aid in push-off. This is followed by TO, where the controller lengthens the actuator to induce dorsiflexion, thus preventing toe drag. Morris et al. [81] have designed a portable powered ankle-foot orthosis (PPAFO) that can assist pilots with lower-limb neuromuscular impairments. The design uses a four-state FSM controller for loading response; early to mid-stance; late stance to pre-swing; and the swing phase. In each state, a fixed torque is applied. Sasaki et al. [82], [83] have designed a pneumatic lower-limb assistive wearable support without a rigid exoskeleton framework. A simple ON-OFF controller is used for each leg. When the CoP moves from one leg to the other, the controller expels the air in the actuator. When the CoP moves back, the inner pressure is increased. Shamaei et al.'s [84] quasi-passive compliant stance control orthosis (CSCO) combines a stiffness switching structure and a lateral joint. This structure incorporates a spring that is parallel to the joint in the stance state, thus allowing for free rotation during the swing state of the gait cycle. A FSM controller is employed to switch from a high stiffness spring during the stance phase to a low stiffness spring during the swing phase. Shen et al. [85], [86] have developed a lower extremity assistive device (LEAD) for stroke patient rehabilitation. In [85], a FSM controller uses the kinematics of the exoskeleton and the GRF. A fixed assistive torque is applied according to different states. In [86], a similar FSM model is used to vary the stiffness and damping coefficients of an impedance controller. The direction of the assistive torque is also based on the FSM model. Wehner et al. [87] have designed a soft lower-limb exosuit that can enhance the muscle function of healthy individuals. The control system, here, employs a simple ONOFF strategy. The heel strike down means turn ON and the foot step up means OFF. A pre-programmed torque sequence is applied when the exosuit is turned on and each leg has a dependent sequence to ensure symmetry. Mooney et al. [88] have developed a powered ankle exoskeleton to provide large amounts of positive mechanical power during plantar flexion. An ON-OFF control strategy is employed, with a low open loop torque being applied in the ON state. Kanno et al. [89] have designed a pneumatically-driven exoskeleton robot to assist walking. The robot is controlled by a five-state FSM model. Three fixed torque assistive functions are applied in these five states because the states of the left leg are the same as the right. Kim et al. [90], [91] have built a hydraulic actuated lower limb exoskeleton for load carrying tasks. This employs a two-state FSM model called "dual mode control" that controls the stance and swing phases separately. The control strategy for the passive mode is to use bypass valves (a mechanical feature) to let the pilot move quickly and freely. In the active mode, the controller aims to minimize the interaction torque and compensates for gravity and the friction of the exoskeleton. Lim et al.'s [92] Hanyang Exoskeleton Assistive Robot (HEXAR)-CR50 employs a two-state FSM model-based controller. A tracking control law is used to prevent the interaction force from going to zero in the swing state. Ka et al. have developed a hybrid control scheme for their PRMI exoskeleton system that augments human power [93]. The hybrid controller consists of two states: swing state and stance state. In the swing state, the exoskeleton is controlled by a global fast sliding mode controller (GF$\mathrm{SMC}$ ) to increase the stability of the system. In the stance state, the system uses a PD controller with gravity compensation. In [94], a similar control scheme is employed, with the stance state still being controlled by a PD controller. However, the control method for the swing state uses a RBF network-based virtual torque control algorithm with dynamic uncertainty compensation instead. Zhou et al. [95] employ a five-state FSM model-based controller to control a powered bionic knee exoskeleton (BioKEX) for the gait rehabilitation of patients with a knee joint movement disorder. The stiffness, damping coefficient and equilibrium angle of the impedance controller are set to vary according to different walking states. Hyun et al. [96] have developed a HLEXv2 exoskeleton robot to provide walking assistance to the elderly. Based on the estimated gait phases for both legs, an assistive control policy is established as a kind of a leg function finite state machine (FLFSM). The FSM maps the estimated gait phase for the controlled leg to its desired function for walking assistance by considering the estimated gait phases for the contralateral leg. The leg functions assigned by the FLFSM are executed by exerting hip or knee flexion/extension torques that are calculated relative to the local coordinates of the hip joint. Lerner et al. [97] have designed a powered exoskeleton for treating the crouching gait of children with cerebral palsy. A three-state FSM divides the gait cycle into the stance state, the early swing state and the late swing state. Constant torques are applied for different 
states by a PID controller. Outside of walking gait control, Thapa et al. [98] have presented a robotic knee orthosis that can assist with sit-to-stand (STS) motions. STS is essentially a transitional motion from one steady state (sitting) to another steady state (standing). A two-state FSM is therefore employed to provide different assistive torque profiles.

Posture-based torque assistance methods are a function of posture and provide a corresponding assistive torque. Gui et al.'s [99] NAEIES, for instance, was developed for carrying heavy loads. They designed a knee joint assistive torque as a function of human forearm motion because they had observed a relationship between the movement of forearms and knee joints. Cao et al. [100] designed a control system for their ELEBOT that includes a posture recognition subprogram and a torque calculation subprogram. The torque calculation function is not fully explained, but from their analysis it can be seen that the assistive torque has a relationship to the joint angle of the pilot. Asbeck et al. have designed two kinds of soft exosuit and a range of control methods. In [126]-[128], the assistive torque for each joint is designed as a function of the walking cadence. Pre-defined normalized reference force profiles are obtained first of all. Then the controller uses these scaled torque profiles based on the walking cadence, which is estimated on the basis of the heel strike signal. However, this control method is only used for a slower walking pace. In [104], [105], a similar control scheme is employed, with the walking cadence being estimated by assessing the maximum hip flexion angle from a biomechanical point of view. The reference torque profiles are scaled according to the walking cadence, as before. The principal difference is that an iterative controller is used to adjust the offset and magnitude of the torque profiles. By having the controller correcting the torque profiles continuously, the desired force can be achieved without needing a perfect initial state. Deng et al. [106] have designed an active power assistive lower-limb (APAL) exoskeleton to assist with random walking movements. The estimated joint torque of the pilot is calculated using inverse dynamics. The pilot's inverse dynamics are estimated from the torso posture and the joint angle of the exoskeleton. The assistive torque is then the product of the estimated joint torque and a coefficient. Schmidt et al.'s [107] Myosuit provides continuous power augmentation at the hip and knee joints for ADLs. The Myosuit employs an anti-gravity torque assistance strategy by supplying the extension torques at the knee and the hip joints. The assistive torques are calculated according to the posture of the pilot's joints and a compensating resistant force is supplied by adjusting the tendon forces and stiffness.

\subsection{Interaction Force-based Torque Assist}

This coordination strategy is similar to the interaction force-based velocity assistance strategy (see Section 3.3). It aims at providing a torque controller that can assist a pilot, whilst causing as little obstruction as possible. The control structure of this strategy is summarized in Fig. 6.

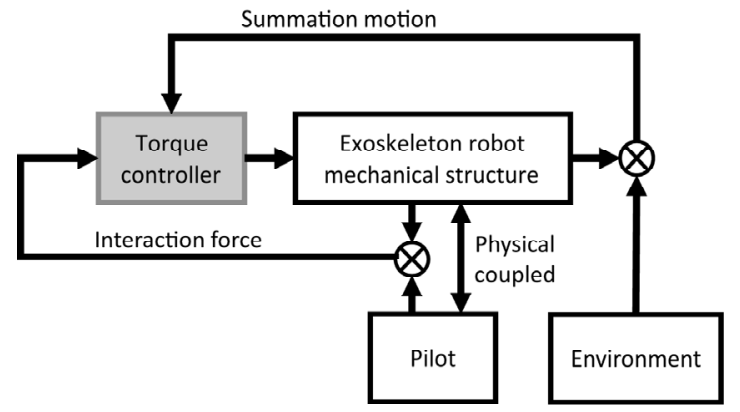

Figure 6. Control structure for interaction force-based torque assistance strategies.

One of the key approaches here is the provision of a virtual admittance controller. Researchers have used this controller to actively change the virtual mechanical impedance of a wearer's limbs while compensating for inertia and friction in the mechanical structure of the exoskeleton. This significantly improves dynamic response and agility of motion. Aguirre-Ollinger et al. have proposed a series of control algorithms to augment walking in this way. The proposed algorithms are implemented in two exoskeleton platforms: the 1-DOF assistive exoskeleton and the Honda stride management assistive device. In [108], the idea of providing negative exoskeleton damping to compensate for limb inertia is proposed, as a kind of active impedance control. In [109], [110], a controller is presented that can generate a negative inertia drive. This control strategy not only compensates for inertia in the mechanical structure of the exoskeleton, but also compensates for inertia in the user's limbs. However, this controller is not stable without effort on the part of the user. Experiments indicate that the coupling system can be made stable if the pilot increases the rhythm of their leg swing in the loop.

Some methods use active impedance to generate adaptive compliance. In [106]-[108], an active impedance control strategy is proposed that is based on a coupled human-exoskeleton model. The main idea, here, is to make the admittance of the coupled system close to the admittance of the lower limb. This control strategy is called "integral admittance (IA) shaping control". The exoskeleton robot provides active impedance to decrease the need for effort on the part of the pilot. Kardan et al.'s [114], [115] FUM-Physio is a single-DOF SEA-powered exoskeleton robot for automating knee therapy. They use the concept of shaping the apparent IA proposed by Nagarajan et al. to develop a novel output feedback assistive control (OFAC) algorithm to control the FUM-Physio. This controller uses the advantages of the SEA actuator and simplifies the IA controller. Huo et al.'s [116] E-ROWA exoskeleton robot can assist STS movements. Its active impedance controller (AIC) is constructed around a state observer and a desired impedance model. The state observer is used to estimate the joint torque of the pilot. The desired impedance model can be adapted to reflect an accurate estimate of the human torque.

The adaptive impedance control method is used in a number of robotic systems. Tran et al. [117] use a fuzzy-based adaptive impedance controller in their PRMI 
lower-limb exoskeleton system, having discovered a relationship between angular frequency and viscous impedance. The inertial and stiffness parameters of the fuzzy controller are empirically chosen in advance. PerezIbarra et al. [118] have developed a simple PD impedance controller with controllable torsional stiffness and damping and programmable reference positions to control a back-drivable ankle robot (Anklebot) that is used for poststroke rehabilitation of ankle joints. To provide adaptive impedance control, the stiffness parameters of the controller are calculated Online from the patient's estimated stiffness using two methods: complementary adaptive control and optimal adaptive control. Liu et al. [119] have designed a two-level control strategy to encourage pilots to perform their best using adaptive compliance in a PMAs-driven ankle rehabilitation exoskeleton robot. The higher level is a task space adaptive admittance controller that considers the pilot's ability to adjust the admittance parameters. The lower level is a joint space adaptive compliance controller that adjusts the compliance level of the PMAs according to the active contribution from the pilot.

The main idea of zero torque control (making the interaction force equal to zero) is to compensate for mechanical friction, inertia, gravity, etc. and to minimize the extent to which the exoskeleton robot might hinder a pilot's movements. Martelli et al. [120] developed a zerotorque cascade controller with a 2-pole 2-zero control loop outside a velocity control loop. The controller was used in a pelvis exoskeleton robot to investigate the behaviour of healthy pilots when wearing it. Their experiments showed that, during uninterrupted walking, the exoskeleton could affect the pilot's movements, obliging the pilot to manage unexpected slip-like perturbations. Haider et al. [121] have presented a "harmonized controller" to assist pilots by eliminating the interaction forces. For simplicity, a PID controller was employed in this approach. Claros et al. [122] have proposed a dual zero force controller for a powered knee orthosis driven by active back-drivability on a geared electromagnetic motor. The front and rear interaction forces can be sensed inside the actuation system. A P controller is employed in this system to bring the interaction forces to zero in both directions. Saccares et al.'s [123] iT-Knee can assist the motion of lower limbs when carrying heavy loads. The iT-Knee innovatively offers one active DOF (knee flexion/extension) that is driven by a SEA actuator and five passive DOFs. A zero torque control law was also implemented to ensure unconstrained knee motion across the whole gait cycle. Bacek et al. [124] have developed a PID-based zero torque controller for their BioMot gait exoskeleton robot that is driven by MACCEPA actuators. The zero torque controller is actually a position controller as the torque of the MACCEPAs can be directly estimated from the joint angle, in much the same way as a SEA. Kim et al. [125] have proposed a control method called "model-inverse time delay control" (MiTDC) for lower-extremity exoskeleton systems driven by a SEA. MiTDC improves upon TDC by adding in virtual referencing. The reference position of the SEA model can be determined according to the measured intention of the pilot and the reference torque trajectory.
The sensitive amplification control (SAC) method is a kind of zero torque control method that is widely used for load-carrying exoskeleton robots. Kazerooni and Huang et al. [144]-[146] are renowned for their design of the human augmentation exoskeleton robot BLEEX. To simplify the control, they gave the BLEEX three distinct states that manifest three different dynamic models: single support; double support; and double support with redundancy. In [128], the control structure for the whole walking phase employs a SAC method. This method uses the inverse dynamics of the exoskeleton as positive feedback, making the loop gain of the exoskeleton nearly uniform. The control law was defined as follows:

$$
T=\hat{P}(\theta)+\left(1-\alpha^{-1}\right)[\hat{M}(\theta) \ddot{\theta}+\hat{C}(\theta, \dot{\theta}) \dot{\theta}]
$$

where $T$ is the applied torque; $\theta$ is the angle of joint; $\hat{C}(\theta, \dot{\theta}), \hat{P}(\theta)$ and $\hat{M}(\theta)$ are the estimates of the Coriolis matrix, the gravity vector and the inertia matrix, respectively; and $\alpha$ is the amplification number. In [126], the stance phase uses a proportional position controller. In the swing phase, they use the SAC. Huang et al. [129] have extended the traditional SAC algorithm into an adaptive SAC (ASAC). Their adaptive SAC strategy can learn the control parameters of the powered exoskeleton. Inspired by the SAC algorithm, this model-based controller has an added sensitivity factor.

Virtual model control (VMC) is more or less the same as the adaptive reference trajectory assistance method discussed in the position assistance section. VMC is mainly used to increase the participation of the wearer during the robot-assisted rehabilitation process. van der Kooij et al. [130] designed a VMC to modify the torques exerted by the exoskeleton robot LOPES. The virtual force here was defined as one that partially counteracts the gravitational force. Unluhisarcikli et al. [131] designed the ANdROS exoskeleton for gait rehabilitation. Its controller generates virtual torque fields at the knee joint. The torque field controller is based on the impedance control and can enable deviations from the reference trajectory. Winfree et al. have proposed two control strategies for their ALEX II system. In [132], a zero-torque controller is first of all employed to make sure that the exoskeleton robot follows the pilot's movements. Then a reference torque profile with a virtual spring model is applied during gait rehabilitation training. The torque profile is based upon a Cartesian coordinate system. The controller can guide a subject's leg back to the desired trajectory. In [133], they employed a hierarchical control architecture. Here, a high-level controller allows a therapist to set a force field (either divergent or convergent) around a target trajectory. A lowlevel controller is then responsible for accurately tracking the desired torque.

\subsection{Gait Feature Estimation-based Torque Assistance}

This control strategy allows an exoskeleton robot to coordinate with a pilot by estimating features of the pilot's 


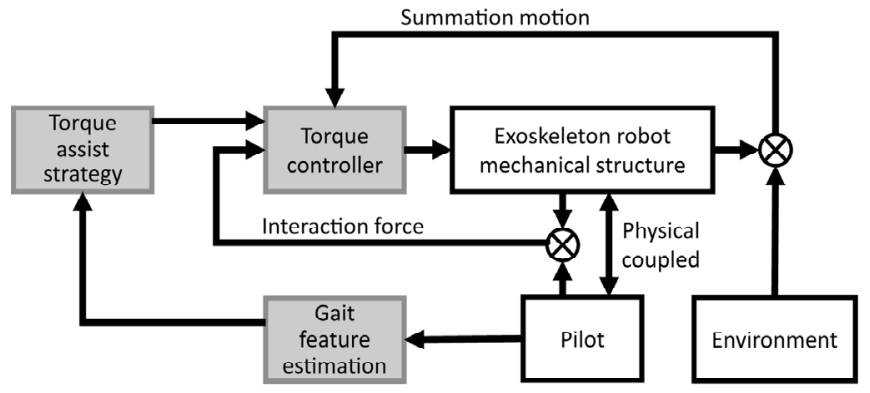

Figure 7. Control structure for the gait feature estimationbased torque assistance strategy.

gait such as cadence and phase. It is based on the hypothesis that the pilot will perform rhythmic movements. The control structure is summarized in Fig. 7. The structure is primarily focused on solving two problems: how to estimate and extract the gait features; and how to design the torque assistance strategy on the basis of those features. Various approaches to gait feature estimation and torque assistance are discussed here according to their use in different systems.

Lenzi et al. [134] estimate walking cadence and gait phase using an adaptive frequency oscillator (AFO) with resistive foot pressure sensors. The gait phase is considered to be a continuous phase normalized in the range $0 \%-100 \%$. This is different from the gait events we have discussed previously. An assistive controller based on the normalized gait phase was designed for the ALEX II gait trainer. The torque assistance strategy took the Winter's CGA data as a function of the gait phase and stepping cadence. Three torque profiles were developed for a slow, normal and high cadence. In [135], the gait phase is also estimated by an AFO. The torque assistance strategy is a function of the normalized gait phase. It has two modes: a learning mode and an assistive mode. The learning mode uses weighted linear regression to obtain specific muscle activity patterns during cyclic movements in the EMG envelope. The learned torque profiles are then encoded for use in the assistive mode. In the assistive mode, the assistive torque is calculated from the learned torque profile and the estimated gait phase and is tracked by an admittance control model. In [136], Zanotto et al. set out the design of an adaptive "assist as needed" controller for the ALEX III bilateral exoskeleton. This controller aims to improve the recovery of gait symmetry. They used the gait phase and joint angles of a healthy leg to estimate the desired torque for an impaired leg. Non-linear filters were employed to learn the foot trajectories of a healthy leg in a task space. Then a VMC-based controller provided low-level control to drive the exoskeleton. Petric et al. [137] compared three different control methods for a squatting assist exoskeleton: a gravity compensation approach; a reference trajectory assisted approach; and an AFO-based assisted torque approach. The AFO-based approach learned the periodicity of the squatting motion and provided a torque assistance law based on the learned motion. Experiments have shown that an AFO-based control method can adaptively and perfectly assist any participant but without offering any obvious advantage over the other two methods. van Dijk et al. [138] have tried employing an AFO in combination with kernel-based non-linear filters to control the Lopes robot. In the Lopes system, the SEAs were originally PI controlled. Phase estimation during the walking phase is provided by the AFO. The phase estimation is then used as input for dynamic compensation and feed-forward elements. The dynamic compensation is used to compensate for friction, gravity and inertia in the exoskeleton so as to provide an accurate torque output. For feed-forward velocity learning, the feed-forward signal is obtained with a non-linear filter. The cyclic pattern of the walking provides a means of estimating the feed-forward signal. The RMS of the torque tracking error here showed a large improvement when compared to the results of a standard PI controller. Kerestes et al. [139] have designed a pneumatically-actuated power exoskeleton to enhance walking and running. A phase portrait based on oscillating terms is employed to determine the direction of the torque and to adjust the assistive frequency of the exoskeleton, adding positive power to the human-machine coupling. Seo et al. [140] have developed an assistive torque control strategy based on a particularly shaped adaptive oscillator (PSAO) for hip exoskeletons. The PSAO is an AFO variant that can be used to track the gait phase too. The control scheme is similar to [134], with the torque profile being normalized according to the body weight. The torque profile is collected as a function of the gait phase by interpolating predefined torque patterns for various speeds and ground tilts. Olivier et al. [141] designed the HiBSO to assist with hip flexion/extension movements during walking. Two different control methods were employed: a zerotorque mode and a gait phase-based assistance mode. The assistance mode is similar to [134] with the assistive torque being tracked by the zero-torque mode. Sugar et al. [142] have developed the HeSA (Hip Exoskeleton for Superior Assistance) to assist with hip flexion and extension as well. The control structure of this system is similar to [139]. It employs a phase portrait-based oscillator to assist with hip movements in the sagittal plane. van Dijk et al. [143] have designed a gait phase-based torque assist control structure for the Achilles ankle exoskeleton to provide push-off assistance for healthy subjects during walking. The gait phase is estimated by an AFO, then a low-pass filter is used to prevent abrupt changes in the gait phase estimation. A torque profile function offers push-off torque according to the phase. A PI-based motor current regulator is used to provide low-level control. Garate et al. [144,145] have presented a gait phase estimation-based control structure that is intended to help pilots in complicated terrain. The gait phase is estimated by an AFO whilst, at the same time, the walking intention is estimated to determine the terrain. A motor primitive-based torque assistance method is also incorporated here. The motor primitive profile is defined by clinical walking data as a function of the gait phase and the primitives of muscle tendon phases are then combined using appropriate weights. Different walking states correspond to different weights. The assistive torque is estimated using the muscle-derived primitives and a Hill 
Summation motion

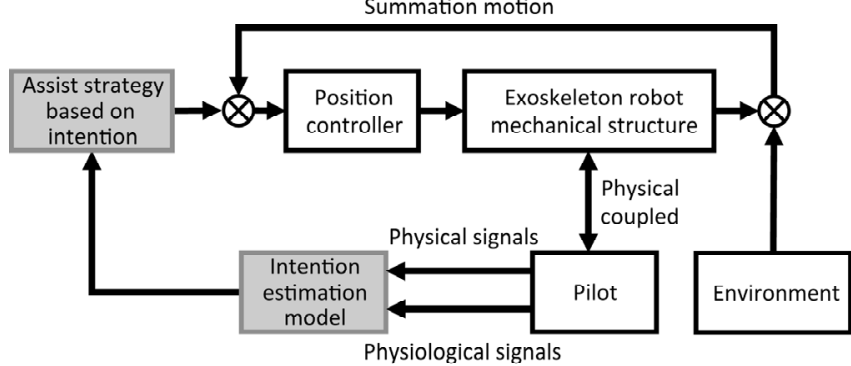

Figure 8. Control structure for physiological intention estimation-based position assistance strategy.

muscle model. The overall aim of this strategy is to adjust different torques smoothly.

\subsection{Physiological Intention Estimation-based Position Assistance}

This strategy estimates a pilot's intentions on the basis of physiological signals and provides coordination using these estimated intentions. The advantage of this strategy is that the physiological signals inevitably precede physical motion. Even when a pilot's limb is not free to move, the intention can be obtained using this approach, setting it apart from position assistance strategies that require physical input. This control structure is summarized in Fig. 8.

One approach to intention estimation is classification. Kasaoka and Sankai [146] have proposed stepping up power assistance according to different step heights using a method that combines the phase sequence and estimated reference input from the EMG. The fixed reference input for the right hip joint during the leg's raising state is altered on the basis of the estimated reference input calculated from the EMG. The bend angle of the joint can also be estimated using the EMG. Contreras-Vidal et al. [147], [148] have employed a brain-machine interface (BMI) to control the NeuroRex exoskeleton robot. In [147], they present the relationship between scalp-recorded EEG activity and functional BOLD signal magnetic resonance imaging (fMRI) activation and de-activation of the cortical and subcortical areas during desired movements. This is proposed as a way of decoding the motor intent of a pilot. These command signals could then be used as input to the Rex so that it could walk autonomously. In [148], the visual cue driven EEG-derived intent of a pilot was identified through a series of binary classifications. There were three classes: walk forward; turn left; and turn right. The first classifications were used to distinguish between walking forward or changing direction. The second set of classifications was used to determine whether to turn left or right once a change in direction had been identified in the first classification phase. The Rex can walk autonomously according to these states. Gui et al. [149] have proposed a control structure for rehabilitation systems based on an EEG and EMG interface. Here, the EMG signals are used to estimate the joint torque of the pilot. The estimated torque is then used to adjust the degree of assistance through a simple admittance controller. The rehabilitation reference trajectory of the admittance controller is defined by an AFO simulation of a healthy subject walking normally on a treadmill at $1.2 \mathrm{~m} \mathrm{~s}^{-1}$. Using the AFO, the walking frequency of the reference trajectory is made adaptable. Thus, SSVEP-based cognitive human-machine interfaces can be employed to identify a normal walking mode, a walking acceleration mode, a walking deceleration mode and a stop mode.

An alternative method is based on the physiological signal regression. Meng et al. [150], [151] have proposed an admittance controller that can turn actual interaction force tracking into an EMG-based interactive force prediction. EMG-based interactive torque is predicted using a support vector regression (SVR) algorithm. The error between the actual interaction force and the predicted force is used as feedback to the admittance controller. The robot impedance can then be adjusted to patients with different recovery conditions by tuning the impedance model and the stiffness coefficient. Fan et al. [152] have designed an EMG-based controller for an ankle exoskeleton that can be used for ankle joint rehabilitation. A fuzzy-neuro network is used to predict human motion and coordinate the control of the human-robot coupling system. The EMG, joint angle and angular velocity signals are acquired and preprocessed first of all. Locomotion decoding and intention estimation are then acquired by fusing the EMG signal and the proprioceptive feedback. Inverse kinematics and a motion controller are then used to block control the exoskeleton directly.

\subsection{Physiological Intention Estimation-based Velocity Assistance}

The control structure of this coordination strategy is similar to Fig. 8. In [153], Lee et al. present a knee movement assistive system (KAS) with an EMG-based controller. Custom-made muscle stiffness sensors based on the EMG are used to obtain a signal related to the degree of muscle expansion and this signal is used in turn to drive their proposed KAS. A fixed motor velocity is set when the EMG signal and the knee angle reach an upper threshold.

\subsection{Physiological Intention Estimation-based Torque Assistance}

The control structure of this strategy is also similar to Fig. 8. Intention estimation can be based on classification and regression.

When using regression, a direct torque assistance strategy is often employed. Direct torque assistance strategies estimate the joint torque directly according to the physiological signals and use the estimated torque to assist the motion directly. Sankai et al.'s Hybrid Assistive Leg (HAL) series is an independent walking aid for people with gait disorders, the elderly and as a means of augmenting human strength. Numerous control structures have been developed for and implemented in HAL. In [154], the joint torque of the exoskeleton robot is considered to be proportional to the EMG of the pilot's extensor and flexor. 
On the basis of this, assisted torques are calculated according to the EMG signals generated by each action. The joint torques for the knee and hip joints are calculated using the following equations:

$$
\begin{gathered}
\hat{\tau}_{\text {knee }}(t)=K_{1} E_{1}(t)-K_{2} E_{2}(t), \\
\hat{\tau}_{\text {hip }}(t)=K_{3} E_{3}(t)-K_{4} E_{4}(t),
\end{gathered}
$$

where $\hat{\tau}_{\text {knee }}$ and $\hat{\tau}_{\text {hip }}$ are the estimated torque of knee joint and hip joint, respectively; $E_{1}, E_{2}, E_{3}$ and $E_{4}$ are the surface EMG signals measured from the biceps femoris, medial vastus, rectus femoris and gluteus maximus, respectively; and $K_{1}, K_{2}, K_{3}$ and $K_{4}$ are the parameters that relate the preceding EMG signals to torques, respectively. In [154], walking is divided into two phases: a swing phase and a support phase. In the swing phase, the EMG-based feedback controller is used for the hip joint. A torque assistance ratio controller and a proportional controller are used to maintain the assistance ratio at 0.6 . If the reference assistance torque and the previous assistance ratio are equal, then the previous assistance torque is used for the next assistance torque. In [155], a power assistive control structure based on human intention is brought about by mixing two control methods: voluntary control and autonomous control. In the voluntary control process, the HAL generates assistive torque by augmenting the joint torque of the pilot, as calculated from the pilot's bio-electrical signals (BES). The assistive torque for each joint is obtained in a fashion similar to [154]. In the autonomous control process, various kinds of information, such as GRF and joint angles, can be used to offer appropriate physical support via a PD controller. The HAL provides the desired functional movement according to the wearer's body structure, the conditions and the purpose of the locomotion assistance. Fleischer et al. [156] have investigated two control approaches for human motion support using exoskeleton robots. The first method uses a dynamic human body model to control the joint angles. The second method controls the assistive torque directly. Both methods need to estimate the pilot's muscle forces using EMG-based muscle and actuator models. In the first method, the dynamic human body model integrates the muscle forces, joint angles and floor contact events and outputs the desired joint angle for the exoskeleton. In the second method, the estimated muscle forces are converted into joint torque. On the basis of this estimated torque, the assistive torque is calculated by multiplying it by an assistive ratio. Kiguchi et al. [157162] have designed an exoskeleton to help physically disabled or elderly people walk in ADL. In [157], the exoskeleton is controlled according to the EMG signals on the skin surface and the interaction force. A fuzzy-neuro network with $20 \mathrm{IF}-\mathrm{THEN}$ rules is used to enable STS and squatting motions. An evaluation function adapts the weights of the fuzzy-neuro network via a back-propagation learning algorithm. In [158], a muscle-model-oriented EMG-based control method is employed, where the assistive joint torque is defined by a matrix that is related to muscle activity. The matrix is adjusted by a fuzzy-neuro network according to the posture. In [160]-[163], the human-robot coordination control strategy is the same as in [158]. All of these papers are focused upon environment sensing and fall prevention. In [163], Chen et al. examine the feasibility of combining functional electrical stimulation (FES) with exoskeletonbased rehabilitation training. The control strategy here involves two functional models: impedance control and FES control. The impedance control in this system is intended to ensure active compliance. The FES control is used to generate the desired joint torque for the pilot. The desired joint torque for the FES control function is estimated from EMG signals. The assistive torque offered by the FES has the same direction as a predefined trajectory in a task space. The pulse width of the FES is controlled using a three-layered BP neural network. Y. Fan and Y. Yin [164] have designed a progressive rehabilitation strategy using EMG. Four progressive rehabilitation modes are proposed according to the suggestions of a doctor specialized in poststroke rehabilitation: passive flexion/extension $(\mathrm{F} / \mathrm{E})$; active $\mathrm{F} / \mathrm{E}$; passive and active gait training with full body weight support. The passive mode is controlled by a positional PID controller that tracks a predefined reference joint angle trajectory. In the active mode, the assistive force is determined by the EMG. Grazi et al. [165] have designed an active pelvis orthosis for walking assistance. A two-layer hierarchical architecture is developed, where the high-level layer is composed of a gait phase estimator and an EMG-based assistive torque calculator. The gait phase estimator is based on an AFO and foot strike events, as previously discussed. The main purpose of the EMGbased assistive torque calculator is to deliver assistive ankle push-off torque. The assistive torque is proportional to the linear envelope of the EMG raw signals in the gait phase $[20 \% ; 60 \%$ and $70 \% ; 10 \%]$. The low-level control is a 2-pole 2-zero closed loop compensator. Koller et al. [166] have designed a dynamically adaptive proportional EMG controller for robotic ankle exoskeletons that can be used to provide assistance to users and reduce metabolic power during walking. In a traditional proportional EMG controller, the linear envelope of a user's raw soleus EMG would be multiplied by a static mapping gain to calculate the control signal. In this study, the mapping gain was dynamically adjusted by the controller using the following methodology: a maximum search on a stride-by-stride basis was conducted; then the maximum values were used to calculate the mapping gain by which the linear envelope is needed to be multiplied to create the actuation control signal. Shabani et al. [167] have designed an EMG-based assistive torque controller that is similar to [156]. The controller records the EMG signals and converts them to activation functions. Then, the model predicts the torque of a patient's knee joint. After this, the estimated torque is multiplied by the support ratio to support the patient's knee, drawing in part upon his or her own torque for every specific movement. Finally, the desired torque is converted to motor current using a closed loop controller.

The intention switching estimation strategy aims to estimate a pilot's locomotion intention through biological signals and to switch between assistive methods in real time. The advantage of this assistive strategy is that a pilot's biological signal is theoretically prior to its realization, 
Table 2

Summarization of Reviewed Articles

\begin{tabular}{|c|c|}
\hline Control strategy & Index of articles \\
\hline $\begin{array}{l}\text { Gait trajectory based } \\
\text { position assistance }\end{array}$ & $\begin{array}{l}{[14],[15],[16],[17],[18]-[20],[21],[22],[23],[24],[25],[26],} \\
{[27],[28],[29],[30],[31],[32],[33],[34],[35],[36],[37],[38],} \\
{[39],[40],[41],[42],[43]-[45],[46],[47],[48],[49],[50],} \\
{[51],[52],[53],[54],[55],[56],[57],[58]}\end{array}$ \\
\hline $\begin{array}{l}\text { Posture estimation based } \\
\text { position assistance }\end{array}$ & {$[59],[60],[61],[62],[63],[64],[65]$} \\
\hline $\begin{array}{l}\text { Interaction force based } \\
\text { velocity assistance }\end{array}$ & {$[66],[67],[68],[69],[70]$} \\
\hline $\begin{array}{l}\text { Posture estimation based } \\
\text { torque assistance }\end{array}$ & $\begin{array}{l}{[71],[72],[73],[74]-[76],[77],[78],[79],[80],[81],[82],} \\
{[83],[84],[85],[86],[87],[88],[89],[90],[91],[92],} \\
{[93],[94],[95],[96],[97],[98],[99],[100]} \\
{[101]-[103],[104],[105],[106],[107]}\end{array}$ \\
\hline $\begin{array}{l}\text { Interaction force based } \\
\text { torque assistance }\end{array}$ & $\begin{array}{l}{[108],[109],[110],[111]-[113],[114],[115],[116],[117],[118],[119],} \\
{[120],[121],[122],[123],[124],[125],[126]-[128],} \\
{[129],[130],[131],[132],[133]}\end{array}$ \\
\hline $\begin{array}{l}\text { Gait feature estimation based } \\
\text { torque assistance }\end{array}$ & $\begin{array}{l}{[134],[135],[136],[137],[138],[139],} \\
{[140],[141],[142],[143],[144],[145]}\end{array}$ \\
\hline $\begin{array}{l}\text { Physiological intention estimation } \\
\text { based position assistance }\end{array}$ & {$[146],[147],[148],[149],[150],[151],[152]$} \\
\hline $\begin{array}{l}\text { Physiological intention estimation } \\
\text { based velocity assistance }\end{array}$ & {$[153]$} \\
\hline $\begin{array}{l}\text { Physiological intention estimation } \\
\text { based torque assistance }\end{array}$ & $\begin{array}{l}{[154],[155],[156],[157]-[162],[163],[164],[165],} \\
{[166],[167],[168],[169],[170],[171]}\end{array}$ \\
\hline
\end{tabular}

so the assistive strategy should not hinder the motion of the pilot. Mavroidis et al. [168] have designed a biofeedback knee device for multiple rehabilitation stages that uses various treatment techniques. The idea here is that when the pilot wants to move but the muscles are too weak, electrical stimulation is provided to assist the pilot. This can be paraphrased as the EMG of the subject being sensed as ongoing motion, but the changes in joint angles in the exoskeleton come to a stop, at which point electrical stimulation is provided to assist. The electrical stimulation causes the quadriceps to contract, helping the subject to achieve full extension. Kawamoto et al. [169] have designed a single leg version of the HAL to enhance the quality of life for patients with hemiplegia. The control method proposed in this case consists mainly of a command signal and torque generation. The command signal is a binary signal obtained from the bioelectrical signal of the knee flexor. The command signal is triggered when the wearer intends to bend the knee joint. When the HAL action has been triggered, an assistive torque is provided to the patients' knee. The torque is generated by the sum of a constant assistive torque, a viscous torque and a gravity compensating torque. Huang et al. [170] have designed a powered lower-limb orthosis for alleviating the problem of cartilage deterioration, thereby enabling elderly people to ascend and descend ladders. They developed a mode switch control strategy according to EMG-based gait mode identification. In the stance mode, a fuzzy model with concentric muscle contraction was employed to calculate the assistive torque. During the swing mode, a fuzzy model with eccentric muscle contraction was employed. In the free motion mode, the exoskeleton followed the movement of the lower limb without hindering the pilot's manoeuvrability.

Alongside of this, Karavas et al. [171] have developed a tele-impedance-based assistive controller that can be used to control the active impedance of an exoskeleton in real-time according to the stiffness estimated from the EMG of a pilot's leg. 


\section{Conclusion}

We have used the Web of science database to collect and screen 158 articles about control methods for lower-limb exoskeleton robots. These articles were then examined and analysed to provide insights regarding the state-of-the-art for the coordination of lower-limb exoskeleton robots and their human pilots. In particular, we wanted to understand what kind of cooperative control strategy was being used. We believe that investigation of the coordination control strategies used for lower-limb exoskeleton robots should start by determining the inputs and outputs of the controller. Therefore, all the articles were initially divided into their interest in physical inputs or physiological inputs, according to the human motion loop in neurobiology and existing human motion intent detection interfaces. Then, depending on the physical effects of the exoskeleton robot on the human body, the articles were subdivided in terms of position assistance, velocity assistance and torque assistance. Providing torque assistance is the most direct method. Position assistance and velocity assistance eventually register as torque for the wearer. Providing position assistance can prevent a pilot from moving freely. Ideally, neither the wearer nor the external environment should need to change with regard to the motion trajectory in this mode. Providing velocity assistance can make a wearer's angular velocity move at a certain speed during exercise. Unlike position assistance, a wearer can slowly change the position of the joint in the assisted velocity mode, so this stands somewhere between position assistance and torque assistance.

Taking these considerations into account, the article has been divided on the basis of a man-machine coordination perspective. Ultimately, this led to the article being divided into nine categories: gait trajectory-based position assistance (drawing on 45 articles); posture estimationbased position assistance (drawing on 7 articles); interaction force-based velocity assistance (drawing on 5 articles); posture estimation-based torque assistance (drawing on 37 articles); interaction force-based torque assistance (drawing on 26 articles); gait feature estimation-based torque assistance (drawing on 12 articles); physiological intention estimation-based position assistance (drawing on 7 articles); physiological intention estimation-based velocity assistance (drawing on 1 article); and physiological intention estimation-based torque assistance (drawing on 18 articles). All the reviewed articles are summarized in Table 2. Each coordination strategy was summarized in terms of its control structures, and the article was discussed in relation to how it focused on these control structures. The organization of this paper provides a technology index for each control strategy.

\section{Acknowledgement}

The work described in this paper is partially supported by the NSFC-Shenzhen Robotics Research Center Project (U1613219), the Shenzhen Fundamental Research and Discipline Layout project (JCYJ20150925163244742) and Shenzhen Technology Project(JSGG20160301160759264).

\section{References}

[1] A. Zoss, H. Kazerooni, and A. Chu, Biomechanical design of theBerkeley lower extremity exoskeleton (BLEEX), IEEE/ASME Transactions on Mechatronics, 11(2), 2006, $128-138$.

[2] K. Amundson, J. Raade, N. Harding, and H. Kazerooni, Hybrid hydraulic-electric power unit for field and service robots, Proc. IEEE/RSJ Conf. on Intelligent Robots and Systems, Edmonton, Alta., Canada, 2005, 3453-3458.

[3] R. Steger, S. Kim, and H. Kazerooni, Control scheme and networked control architecture for the Berkeley lower extremity exoskeleton (BLEEX), Proc. IEEE Conf. on Robotics and Automation, Orlando, FL, USA, 2006, 3469-3476.

[4] H. Kazerooni, N. Harding, and R. Angold, Low extremity exoskeleton, Int. Patent WO 2006/078 871A2, 2006.

[5] H. Kazerooni, N. Harding, R. Angold, K. Amundson, J. W. Burns, and A. Zoss, Wearable material handling system, Int. Patent WO 2010/101 595A1, 2010.

[6] [Online]. Available: http://www.rb3d.com/en/exo/ (accessed on Sep. 2015).

[7] S. Yoshiyuki, HAL: Hybrid assistive limb based on cybernics, Robotics research (Berlin, Heidelberg: Springer, 2010), 25-34.

[8] Y. Tingfang, C. Marco, O.C. Maria, and V. Nicola, Review of assistive strategies in powered lower-limb orthoses and exoskeletons, Robotics and Autonomous Systems, 64,2015, 120-136.

[9] N. Domen and R. Robert, A survey of sensor fusion methods in wearable robotics, Robotics and Autonomous Systems, 73, $2015,155-170$.

[10] M. Wei, L. Quan, Z. Zude, A. Qingsong, S. Bo, and X.S. Shane, Recent development of mechanisms and control strategies for robot-assisted lower limb rehabilitation, Mechatronics, 31, 2015, 132-145.

[11] M.R. Tucker, O. Jeremy, P. Anna, B. Hannes, B. Mohamed, L. Olivier, D.R.M. José, R. Robert, V. Heike, and G. Roger, Control strategies for active lower extremity prosthetics and orthotics: a review, Journal of Neuroengineering and Rehabilitation, 12(1), 2015, 1.

[12] V.A. Joshua and X.S. Quan, Towards compliant and wearable robotic orthoses: A review of current and emerging actuator technologies, Medical Engineering 83 Physics, 4, 2016, 317325.

[13] W. Michael, G. Martin, C. Oliver, R. Stephan, and B. Philipp, Active lower limb prosthetics: a systematic review of design issues and solutions, Biomedical Engineering Online, 15(3), 2016, 140

[14] S.K. Banala, S.H. Kim, S.K. Agrawal, and J.P. Scholz, Robot assisted gait training with active leg exoskeleton (ALEX), IEEE Transactions on Neural Systems and Rehabilitation Engineering, 17(1), 2009, 2-8.

[15] S.K. Banala, S.K. Agrawal, S.H. Kim, and J.P. Scholz, Novel gait adaptation and neuromotor training results using an active leg exoskeleton, IEEE/ASME Transactions on Mechatronics, 15(2), 2010, 216-225.

[16] X. Jin, X. Cui, and S.K. Agrawal, Design of a cable-driven active leg exoskeleton (C-ALEX) and gait training experiments with human subjects, Proc. IEEE Conf. on Robotics and Automation, Seattle, WA, USA, 2015, 5578-5583.

[17] G.S. Heo, S.-R. Lee, M.K. Kwak, C.W. Park, G. Kim, and C.-Y. Lee, Motion control of bicycle-riding exoskeleton robot with interactive force analysis, International Journal of Precision Engineering and Manufacturing, 16(7), 1631-1637.

[18] P. Beyl, M. Van Damme, P. Cherelle, and D. Lefeber, Safe and compliant guidance in robot-assisted gait rehabilitation using proxy-based sliding mode control, Proc. IEEE Conf. on Rehabilitation Robotics, Kyoto, Japan, 2009, 321-326.

[19] P. Beyl, K. Knaepen, S. Duerinck, M. Van Damme, B. Vanderborght, R. Meeusen, and D. Lefeber, Safe and compliant guidance by a powered knee exoskeleton for robotassisted rehabilitation of gait, Advanced Robotics, 25(5), 2011, 513-535.

[20] K. Knaepen, P. Beyl, S. Duerinck, F. Hagman, D. Lefeber, and R. Meeusen, Human-robot interaction: kinematics and muscle activity inside a powered compliant knee exoskeleton, 
IEEE Transactions on Neural Systems and Rehabilitation Engineering, 22(6), 2014, 1128-1137.

[21] A.I.A. Ahmed, H. Cheng, X. Lin, Z.M.E. Elhassan, and M. Omer, On-line walking speed control in human-powered exoskeleton systems, Int. Conf. on Communication, Control, Computing and Electronics Engineering, Khartoum, Sudan, 2017, 1-7.

[22] A.I.A. Ahmed, C. Hong, L. Zhang, M. Omer, and X. Lin, On-line walking speed control in human-powered exoskeleton systems based on dual reaction force sensors, Journal of Intelligent and Robotic Systems, 87(1), 2017, 59-80.

[23] J. Hu, Z.-G. Hou, Y. Chen, L. Peng, and L. Peng, Task-oriented active training based on adaptive impedance control with iLeg-a horizontal exoskeleton for lower limb rehabilitation, Proc. IEEE Conf. on Robotics and Biomimetics, Shenzhen, China, 2013, 2025-2030.

[24] R. Lopez, H. Aguilar-Sierra, S. Salazar, J. Torres, and R. Lozano, Adaptive control for passive kinesiotherapy ELLTIO, Proc. IEEE Conf. on Advanced Robotics, Montevideo, Uruguay, 2013, 1-6.

[25] B. Ugurlu, H. Oshima, and T. Narikiyo, Lower body exoskeleton-supported compliant bipedal walking for paraplegics: how to reduce upper body effort?, Proc. IEEE Conf. on Robotics and Automation, Hong Kong, China, 2014, 1354-1360.

[26] P.K. Jamwal, S. Hussain, M.H. Ghayesh, and S.V. Rogozina, Impedance control of an intrinsically compliant parallel ankle rehabilitation robot, IEEE Transactions on Industrial Electronics, 63(6), 2016, 3638-3647.

[27] P.K. Jamwal, S. Hussain, M.H. Ghayesh, and S.V. Rogozina, Adaptive impedance control of parallel ankle rehabilitation robot, Journal of Dynamic Systems Measurement and Control-Transactions of the ASME, 139(11), 2017, 1608.

[28] H. Rifai, M.S. Ben Abdessalem, A. Chemori, S. Mohammed, and Y. Amirat, Augmented L1 adaptive control of an actuated knee joint exoskeleton: from design to real-time experiments, Proc. IEEE Conf. on Robotics and Automation, Stockholm, Sweden, 2016, 5708-5714.

[29] H. Rifai, S. Mohammed, K. Djouani, and Y. Amirat, Toward lower limbs functional rehabilitation through a kneejoint exoskeleton, IEEE Transactions on Control Systems Technology, 25(2), 2017, 712-719.

[30] W.M. dos Santos, G.A.P. Caurin, and A.A.G. Siqueira, Design and control of an active knee orthosis driven by a rotary series elastic actuator, Control Engineering Practice, 58, 2017, 307-318.

[31] X. Li, Y. Pan, G. Chen, and H. Yu, Multi-modal control scheme for rehabilitation robotic exoskeletons, International Journal of Robotics Research, 36(5-7), 2017, 759-777.

[32] G. Colombo, M. Joerg, R. Schreier, and V. Dietz, Treadmill training of paraplegic patients using a robotic orthosis, Journal of Rehabilitation Research and Development, 37(6), 2000, 693-700.

[33] B. Chen, X. Zhao, H. Ma, L. Qin, and W.-H. Liao, Design and characterization of a magneto-rheological series elastic actuator for a lower extremity exoskeleton, Smart Materials and Structures, 26(10), 2017, 105008.

[34] Y. Long, Z.-J. Du, W. Wang, and W. Dong, Development of a wearable exoskeleton rehabilitation system based on hybrid control mode, International Journal of Advanced Robotic Systems, 13, 2016, 1729881416664847.

[35] W.Y.-W. Tung, M. McKinley, M.V. Pillai, J. Reid, and H. Kazerooni, Design of a minimally actuated medical exoskeleton with mechanical swing-phase gait generation and sit-stand assistance, ASME Conf. on Dynamic Systems and Control, Palo Alto, California, USA, 2013, 4-13.

[36] W. Banchadit, A. Temram, T. Sukwan, P. Owatchaiyapong, and J. Suthakorn, Design and implementation of a new motorized-mechanical exoskeleton based on CGA patternized control, Proc. IEEE Conf. on Robotics and Biomimetics, Guangzhou, China, 2012, 1668-1673.

[37] D.A. Winter, Anthropometry, in Biomechanics and Motor Control of Human Movement, Chapter 4, 2009, 51-74.

[38] S. Tanabe, S. Hirano, and E. Saitoh, Wearable Power-Assist Locomotor (WPAL) for supporting upright walking in persons with paraplegia, Neurorehabilitation, 33(1), 2013, 99-106.
[39] K.H. Low, X. Liu, and H. Yu, Development of NTU wearable exoskeleton system for assistive technologies, Proc. IEEE Conf. on Mechatronics and Automation, Niagara Falls, Ont., Canada, 2005, 1099-1106.

[40] K.H. Low, X. Liu, C.H. Goh, and H. Yu, Locomotive control of a wearable lower exoskeleton for walking enhancement, Journal of Vibration and Control, 12(12), 2006, 1311-1336.

[41] W. Kim, S. Lee, M. Kang, J. Han, and C. Han, Energy-efficient gait pattern generation of the powered robotic exoskeleton using DME, Proc. IEEE/RSJ Conf. on Intelligent Robots and Systems, Taipei, Taiwan, 2010, 2475-2480.

[42] S. Lee, W. Kim, M. Kang, J. Han, and C. Han, Optimal gait pattern generation for powered robotic exoskeleton and verification of its feasibility, Proc. Int. Symposium in Robot and Human Interactive Communication, Viareggio, Italy, 2010, 500-505.

[43] D. Sanz-Merodio, M. Cestari, J. Carlos Arevalo, and E. Garcia, A lower-limb exoskeleton for gait assistance in quadriplegia, Proc. IEEE/RSJ Conf. on Robotics and Biomimetics, Guangzhou, China, 2012, 122-127.

[44] D. Sanz-Merodio, M. Cestari, J.C. Arevalo, X.A. Carrillo, and E. Garcia, Generation and control of adaptive gaits in lower-limb exoskeletons for motion assistance, Advanced Robotics, 28(5), 2014, 329-338.

[45] D. Sanz-Merodio, J. Sancho, M. Perez, and E. Garcia, Control architecture of the ATLAS 2020 lower limb active orthosis, Advances in Cooperative Robotics, 2017, 860-868.

[46] N. Trung, T. Komeda, T. Miyoshi, and L. Ota, The powered gait training system using feedback from own walking information, Issnip Conf. on Biosignals and Biorobotics, Rio de Janerio, Brazil, 2013, 239-243.

[47] Y. Hasegawa and K. Nakayama, Finger-mounted walk controller of powered exoskeleton for paraplegic patient's walk, World Automation Congress, Waikoloa, HI, USA, 2014, 400405.

[48] M. Li, Z. Yuan, X. Wang, and Y. Hasegawa, Electric stimulation and cooperative control for paraplegic patient wearing an exoskeleton, Robotics and Autonomous Systems, 98, 2017, 204-212.

[49] T. Kagawa, H. Ishikawa, T. Kato, C. Sung, and Y. Uno, Optimization-based motion planning in joint space for walking assistance with wearable robot, IEEE Transactions on Robotics, 31(2), 2015, 415-424.

[50] R. Griffin, T. Cobb, T. Craig, M. Daniel, N. van Dijk, J. Gines, K. Kramer, S. Shah, O. Siebinga, J. Smith, and P. Neuhaus, Stepping forward with exoskeletons team IHMC's design and approach in the 2016 Cybathlon, IEEE Robotics and Automation Magazine, 24(4), 2017, 66-74.

[51] K. Kamali, A.A. Akbari, and A. Akbarzadeh, Trajectory generation and control of a knee exoskeleton based on dynamic movement primitives for sit-to-stand assistance, Advanced Robotics, 30(13), 2016, 846-860.

[52] D.J. Reinkensmeyer, D. Aoyagi, J.L. Emken, J.A. Galvez, W. Ichinose, G. Kerdanyan, S. Maneekobkunwong, K. Minakata, J.A. Nessler, R. Weber, R.R. Roy, R. de Leon, J.E. Bobrow, S.J. Harkema, and V.R. Edgerton, Tools for understanding and optimizing robotic gait training, Journal of Rehabilitation Research and Development, 43(5), 657-670.

[53] H.K. Kwa, J.H. Noorden, M. Missel, T. Craig, J.E. Pratt, and P.D. Neuhaus, Development of the IHMC mobility assist exoskeleton, Proc. IEEE Conf. on Robotics and Automation, Kobe, Japan, 2009, 1349-1355.

[54] P.D. Neuhaus, J.H. Noorden, T.J. Craig, T. Torres, J. Kirschbaum, and J.E. Pratt, Design and evaluation of Mina: a robotic orthosis for paraplegics, Proc. IEEE Conf. on Rehabilitation Robotics, Zurich, Switzerland, 2011, 1-8.

[55] U. Lugris, J. Carlin, A. Luaces, and J. Cuadrado, Consideration of assistive devices in the gait analysis of spinal cord-injured subjects, ASME Conf. on Multibody Systems, Nonlinear Dynamics, and Control, Portland, Oregon, USA, 2014, 9-18.

[56] Y. Yang, C. Yang, K.-M. Lee, and H. Yu, Model-based fuzzy adaptation for control of a lower extremity rehabilitation exoskeleton, Proc. IEEE/ASME Conf. on Advanced Intelligent Mechatronics, Singapore, 2009, 350. 
[57] J.-F. Zhang, Y.-M. Dong, C.-J. Yang, Y. Geng, Y. Chen, and Y. Yang, 5-Link model based gait trajectory adaption control strategies of the gait rehabilitation exoskeleton for post-stroke patients, Mechatronics, 20(3), 2010, 368-376.

[58] A. Duschau-Wicke, J. Von Zitzewitz, L. Luenenburger, and R. Riener, Patient-driven cooperative gait training with the rehabilitation robot lokomat, Proc. Int. Fed. European Conf. on Medical and Biological Engineering, ETH Zurich, Zurich, Switzerland, 2009, 1616-1619.

[59] M. Talaty, A. Esquenazi, and J.E. Briceno, Differentiating ability in users of the ReWalk(TM) powered exoskeleton: an analysis of walking kinematics, Proc. IEEE Conf. on Rehabilitation Robotics, Seattle, WA, USA, 2013, 1-5.

[60] K.A. Strausser, T.A. Swift, A.B. Zoss, and H. Kazerooni, Prototype medical exoskeleton for paraplegic mobility: first experimental results, Proc. ASME Conf. on Dynamic Systems and Control Conference, Cambridge, Massachusetts, USA, 2010, 453-458.

[61] H.A. Quintero, R.J. Farris, and M. Goldfarb, Control and implementation of a powered lower limb orthosis to aid walking in paraplegic individuals, Proc. IEEE Conf. on Rehabilitation Robotics, Zurich, Switzerland, 2011, 1-6.

[62] K.A. Strausser and H. Kazerooni, The development and testing of a human machine interface for a mobile medical exoskeleton, Proc. IEEE/RSJ Conf. on Intelligent Robots and Systems, San Francisco, CA, USA, 2011, 4911-4916.

[63] D. Sanz-Merodio, M. Cestari, J. Carlos Arevalo, and E. Garcia, Control motion approach of a lower limb orthosis to reduce energy consumption, International Journal of Advanced Robotic Systems, 9(6), 2012, 232.

[64] A.J. del-Ama, A. Gil-Agudo, J.L. Pons, and J.C. Moreno, Hybrid FES-robot cooperative control of ambulatory gait rehabilitation exoskeleton, Journal of Neuroengineering and Rehabilitation, 11(4), 2014, 27.

[65] J. Poonsiri, M. Rachagorngij, and W. Charoensuk, Biomechanical based design of an active knee ankle foot orthosis to augment the knee motions, Proc. Int. Conf. on Biomedical Engineering, Fukuoka, 2014, 1-5.

[66] F. Chen, Y. Yu, Y. Ge, J. Sun, and B. Wu, A PAWL for enhancing strength and endurance during walking using interaction force and dynamical information, Porc. IEEE Conf. on Robotics and Biomimetics, Kunming, China, 2006, 654-659.

[67] K. Kong and D. Jeon, Design and control of an exoskeleton for the elderly and patients, IEEE/ASEM Transactions on Mechatronics, 11(4), 2006, 428-432.

[68] B. Weinberg, J. Nikitczuk, S. Patel, B. Patritti, C. Mavroidis, P. Bonato, and P. Canavan, Design, control and human testing of an active knee rehabilitation orthotic device, Proc. IEEE Conf. on Robotics and Automation, Roma, Italy, 2007, 4126-4133.

[69] Q. Wu, X. Wang, F. Du, and X. Zhang, Design and control of a powered hip exoskeleton for walking assistance, International Journal of Advanced Robotic Systems, 12, 2015, 18.

[70] C. Zhang, X. Zang, Z. Leng, H. Yu, J. Zhao, and Y. Zhu, Human-machine force interaction design and control for the HIT load-carrying exoskeleton, Advances in Mechanical Engineering, 8(4), 2016, 1687814016645068.

[71] K. Fujishiro, T. Ariumi, O. Oyama, and T. Yoshimitu, Development of pneumatic assist system for human walk, SICE Annual Conf. Program and Abstracts, 2003, 41.

[72] J. Chen and W.-H. Liao, A leg exoskeleton utilizing a magnetorheological actuator, Proc. IEEE Conf. on Robotics and Biomimetics, Kunming, China, 2006, 824-829.

[73] K.H. Low and Y. Yin, Providing assistance to knee in the design of a portable active orthotic device, Proc. IEEE Conf. on Automation Science and Engineering, Shanghai, China, 2016, 188.

[74] C.J. Walsh, D. Paluska, K. Pasch, W. Grand, A. Valiente, and H. Herr, Development of a lightweight, underactuated exoskeleton for load-carrying augmentation, Proc. IEEE Conf. on Robotics and Automation, Orlando, FL, USA, 2006, 3485.

[75] C.J. Walsh, K. Pasch, and H. Herr, An autonomous, underactuated exoskeleton for load-carrying augmentation, Proc. IEEE Conf. on Intelligent Robots and Systems, Beijing, China, 2006, 1410-1415.
[76] C.J. Walsh, K. Endo, and H. Herr, A quasi-passive leg exoskeleton for load-carrying augmentation, International Journal of Humanoid Robotics, 4(3), 2007, 487-506.

[77] A.M. Oymagil, J.K. Hitt, T. Sugar, and J. Fleeger, Control of a regenerative braking powered ankle foot orthosis, Proc. IEEE Conf. on Rehabilitation Robotics, Noordwijk, Netherlands, 2007, 28.

[78] M. Sugisaka, J. Wang, H. Tsumura, and M. Kataoka, A control method of ankle foot orthosis (AFO) with artificial muscle, SICE Annual Conf., Tokyo, Japan, 2008, 2013-2017.

[79] J.S. Sulzer, R.A. Roiz, M.A. Peshkin, and J.L. Patton, A highly backdrivable, lightweight knee actuator for investigating gait in stroke, IEEE Transactions on Robotics, 25(3), 2009, 539-548.

[80] J. Kim, S. Hwang, R. Sohn, Y. Lee, and Y. Kim, Development of an active ankle foot orthosis to prevent foot drop and toe drag in hemiplegic patients: A preliminary study, Applied Bionics and Biomechanics, 8(3-4), 2011, 377-384.

[81] E.A. Morris, K.A. Shorter, Y. Li, E.T. Hsiao-Wecksler, G.F. Kogler, T. Bretl, and W.K. Durfee, Actuation timing strategies for a portable powered ankle foot orthosis, Proc. ASME Conf. on Dynamic Systems and Control and Bath/ASME Symposium on Fluid Power and Motion Control, Arlington, Virginia, USA, 2011, 807-814.

[82] D. Sasaki, T. Noritsugu, M. Takaiwa, and I.R.S.O. Japan, Development of pneumatic lower limb power assist wear without exoskeleton, Proc. IEEE/RSJ Conf. on Intelligent Robots and Systems, Vilamoura, Portugal, 2012, 1239-1244.

[83] D. Sasaki, T. Noritsugu, and M. Takaiwa, Development of pneumatic lower limb power assist wear driven with wearable air supply system, Proc. IEEE/RSJ Conf. on Intelligent Robots and Systems, Tokyo, Japan, 2013, 4440-4445.

[84] K. Shamaei, P.C. Napolitano, and A.M. Dollar, A quasipassive compliant stance control knee-ankle-foot orthosis, Proc. IEEE Conf. on Rehabilitation Robotics, Seattle, WA, USA, 2013, 1-6.

[85] B. Shen, J. Li, F. Bai, and C.-M. Chew, Development and control of a lower extremity assistive device (LEAD) for gait rehabilitation, Proc. IEEE Conf. on Rehabilitation Robotics, Seattle, WA, USA, 2013, 1-6.

[86] B. Shen, J. Li, and C.-M. Chew, Functional task based assistance during walking for a lower extremity assistive device, Proc. IEEE Conf. on Robotics and Automation, Hong Kong, China, 2014, 246-251.

[87] M. Wehner, B. Quinlivan, P.M. Aubin, E. MartinezVillalpando, M. Baumann, L. Stirling, K. Holt, R. Wood, and C. Walsh, A lightweight soft exosuit for gait assistance, Proc. IEEE Conf. on Robotics and Automation, Karlsruhe, Germany, 2013, 3362-3369.

[88] L.M. Mooney, E.J. Rouse, and H.M. Herr, Autonomous exoskeleton reduces metabolic cost of walking, Proc. IEEE Conf. on Engineering in Medicine and Biology Society, Chicago, IL, USA, 2014, 3065-3068.

[89] T. Kanno, D. Morisaki, R. Miyazaki, G. Endo, and K. Kawashima, A walking assistive device with intention detection using back-driven pneumatic artificial muscles, Proc. IEEE/RAS/EMBS Conf. on Rehabilitation Robotics, Singapore, Singapore, 2015, 565-570.

[90] H. Kim, C. Seo, Y.J. Shin, J. Kim, and Y.S. Kang, Locomotion control strategy of hydraulic lower extremity exoskeleton robot, Proc. IEEE/ASME Conf. on Advanced Intelligent Mechatronics, Busan, South Korea, 2015, 577-582.

[91] H. Kim, Y.J. Shin, and J. Kim, Design and locomotion control of a hydraulic lower extremity exoskeleton for mobility augmentation, Mechatronics, 46, 2017, 32-45.

[92] D. Lim, W. Kim, H. Lee, H. Kim, K. Shin, T. Park, J. Lee, and C. Han, Development of a lower extremity exoskeleton robot with a quasi-anthropomorphic design approach for load carriage, Proc. IEEE/RSJ Conf. on Intelligent Robots and Systems, Hamburg, Germany, 2015, 5345-5350.

[93] D.M. Ka, H. Cheng, T.H. Toan, and Q. Jing, Minimizing human-exoskeleton interaction force using compensation for dynamic uncertainty error with adaptive RBF network, Journal of Intelligent and Robotic Systems, 82(3-4), 2016, 413-433. 
[94] K.D. Mien, C. Hong, T.T. Huu, and J. Qiu, Minimizing human-exoskeleton interaction force by using global fast sliding mode control, International Journal of Control Automation and Systems, 14(4), 2016, 1064-1072.

[95] Z. Zhou, Y. Liao, C. Wang, and Q. Wang, Preliminary evaluation of gait assistance during treadmill walking with a light-weight bionic knee exoskeleton, Proc. IEEE Conf. on Robotics and Biomimetics, Qingdao, China, 2016, 1173-1178.

[96] D.J. Hyun, H. Lim, S. Park, and K. Jung, Development of ankle-less active lower-limb exoskeleton controlled using finite leg function state machine, International Journal of Precision Engineering and Manufacturing, 18(6), 2017, 803-811.

[97] Z.F. Lerner, D.L. Damiano, H.-S. Park, A.J. Gravunder, and T.C. Bulea, A robotic exoskeleton for treatment of crouch gait in children with cerebral palsy: design and initial application, IEEE Transactions on Neural Systems and Rehabilitation Engineering, 25(6), 2017, 650-659.

[98] S. Thapa, H. Zheng, G.F. Kogler, and X. Shen, A robotic knee orthosis for sit-to-stand assistance, Proc. ASME Conf. Dynamic Systems and Control, Minneapolis, Minnesota, USA, 2017, V001T07A004.

[99] L. Gui, Z. Yang, X. Yang, W. Gu, and Y. Zhang, Design and control technique research of exoskeleton suit, Proc. IEEE Conf. on Automation and Logistics, Jinan, China, 2007, 541-546.

[100] H. Cao, Z. Ling, J. Zhu, Y. Wang, and W. Wang, Design frame of a leg exoskeleton for load-carrying augmentation, Proc. IEEE Conf. on Robotics and Biomimetics, Guilin, China, 2009, 426-431.

[101] Y. Ding, I. Galiana, A. Asbeck, B. Quinlivan, S.M.M. De Rossi, and C. Walsh, Multi-joint actuation platform for lower extremity soft exosuits, Proc. IEEE Conf. on Robotics and Automation, Hong Kong, China, 2014, 1327-1334.

[102] A.T. Asbeck, S.M.M. De Rossi, K.G. Holt, and C.J. Walsh, A biologically inspired soft exosuit for walking assistance, International Journal of Robotics Research, 34(6), 2015, 744762.

[103] A.T. Asbeck, K. Schmidt, and C.J. Walsh, Soft exosuit for hip assistance, Robotics and Autonomous Systems, 73, 2015, 102-110.

[104] Y. Ding, I. Galiana, C. Siviy, F.A. Panizzolo, and C. Walsh, IMU-based iterative control for hip extension assistance with a soft exosuit, Proc. IEEE Conf. on Robotics and Automation, Stockholm, Sweden, 2016, 3501-3508.

[105] Y. Ding, F.A. Panizzolo, C. Siviy, P. Malcolm, I. Galiana, K.G. Holt, and C.J. Walsh, Effect of timing of hip extension assistance during loaded walking with a soft exosuit, Journal of Neuroengineering and Rehabilitation, 13, 2016, 87.

[106] J. Deng, P. Wang, M. Li, W. Guo, F. Zha, and X. Wang, Structure design of active power-assist lower limb exoskeleton APAL robot, Advances in Mechanical Engineering, 9(11), 2017, 1687814017735791.

[107] K. Schmidt, J.E. Duarte, M. Grimmer, A. Sancho-Puchades, H. Wei, C.S. Easthope, and R. Riener, The myosuit: biarticular anti-gravity exosuit that reduces hip extensor activity in sitting transfers, Frontiers in Neurorobotics, 11, 2017, 57.

[108] G. Aguirre-Ollinger, J.E. Colgate, M.A. Peshkin, and A. Goswami, A 1-DOF assistive exoskeleton with virtual negative damping: Effects on the kinematic response of the lower limbs, Proc. IEEE/RSJ Conf. on Intelligent Robots and Systems, San Diego, CA, USA, 2007, 1938-1944.

[109] G. Aguirre-Ollinger, J.E. Colgate, M.A. Peshkin, and A. Goswami, Design of an active one-degree-of-freedom lowerlimb exoskeleton with inertia compensation, International Journal of Robotics Research, 30(4), 2011, 486-499.

[110] G. Aguirre-Ollinger, J.E. Colgate, M.A. Peshkin, and A. Goswami, Inertia compensation control of a one-degreeof-freedom exoskeleton for lower-limb assistance: initial experiments, IEEE Transactions on Neural Systems and Rehabilitation Engineering, 20(1), 2012, 68-77.

[111] U. Nagarajan, G. Aguirre-Ollinger, and A. Goswami, Integral admittance shaping for exoskeleton control, Proc. IEEE Conf. on Robotics and Automation, Seattle, WA, USA, 2015, 5641-5648.
[112] G. Aguirre-Ollinger, U. Nagarajan, and A. Goswami, An admittance shaping controller for exoskeleton assistance of the lower extremities, Autonomous Robots, 40(4), 2016, 701-728.

[113] U. Nagarajan, G. Aguirre-Ollinger, and A. Goswami, Integral admittance shaping: A unified framework for active exoskeleton control, Robotics and Autonomous Systems, 75, 2016, 310-324.

[114] I. Kardan and A. Akbarzadeh, Assistive control of a compliantly actuated single axis stage, Int. Conf. on Robotics and Mechatronics, Tehran, Iran, 2016, 313-318.

[115] I. Kardan and A. Akbarzadeh, Output feedback assistive control of single-DOF SEA powered exoskeletons, Industrial Robot-an International Journal, 44(3), 2017, 275-287.

[116] W. Huo, S. Mohammed, Y. Amirat, and K. Kong, Active impedance control of a lower limb exoskeleton to assist sit-to-stand movement, Proc. IEEE Conf. on Robotics and Automation, Stockholm, Sweden, 2016, 3530-3536.

[117] H.-T. Tran, H. Cheng, M.-K. Duong, and H. Zheng, Fuzzybased impedance regulation for control of the coupled humanexoskeleton system, Proc. IEEE Conf. on Robotics and Biomimetics, Bali, Indonesia, 2014, 986-992.

[118] J.C. Perez-Ibarra, A.A.G. Siqueira, and H.I. Krebs, Assistas-needed ankle rehabilitation based on adaptive impedance control, Proc. IEEE/RAS/EMBS Conf. on Rehabilitation Robotics, Singapore, Singapore, 2015, 723-728.

[119] Q. Liu, A. Liu, W. Meng, Q. Ai, and S.Q. Xie, Hierarchical compliance control of a soft ankle rehabilitation robot actuated by pneumatic muscles, Frontiers in Neurorobotics, 11(4), 2017, 64.

[120] D. Martelli, F. Vannetti, M. Cortese, P. Tropea, F. Giovacchini, S. Micera, V. Monaco, and N. Vitiello, The effects on biomechanics of walking and balance recovery in a novel pelvis exoskeleton during zero-torque control, Robotica, 32(8), 2014 $1317-1330$.

[121] U. Haider, I.I. Nyoman, J.L. Coronado, C. Kim, and G.S. Virk, User-centric harmonized control for single joint assistive exoskeletons, International Journal of Advanced Robotic Systems, 13, 2016, 115.

[122] M.J. Claros, R. Soto, J.L. Gordillo, J.L. Pons, and J.L. Contreras-Vidal, Robotic assistance of human motion using active-backdrivability on a geared electromagnetic motor, International Journal of Advanced Robotic Systems, 13, 2016, 40.

[123] L. Saccares, I. Sarakoglou, and N.G. Tsagarakis, iT-Knee: an exoskeleton with ideal torque transmission interface for ergonomic power augmentation, Proc. IEEE/RSJ Conf. on Intelligent Robots and Systems, Daejeon, South Korea, 2016 780-786.

[124] T. Bacek, M. Moltedo, K. Langlois, G.A. Prieto, M.C. Sanchez-Villamanan, J. Gonzalez-Vargas, B. Vanderborght, D. Lefeber, and J.C. Moreno, BioMot exoskeleton - Towards a smart wearable robot for symbiotic human-robot interaction, Proc. IEEE Conf. on Rehabilitation Robotics, London, UK, 2017, 1666-1671.

[125] S. Kim and J. Bae, Force-mode control of rotary series elastic actuators in a lower extremity exoskeleton using modelinverse time delay control, IEEE/ASME Transactions on Mechatronics, 22(3), 2017, 1392-1400.

[126] L. Huang, J.R. Steger, and H. Kazerooni, Hybrid control of the Berkeley lower extremity exoskeleton (BLEEX), The International Journal of Robotics Research, 25(5-6), 2005, $561-573$.

[127] H. Kazerooni, Exoskeletons for human power augmentation, Proc. IEEE/RSJ Conf. on Intelligent Robots and Systems, Edmonton, Alberta, Canada, 2005, 3459-3464.

[128] H. Kazerooni, J.L. Racine, L.H. Huang, and R. Steger, On the control of the Berkeley Lower Extremity Exoskeleton (BLEEX), Proc. IEEE Conf. on Robotics and Automation, Barcelona, Spain, 2005, 4353-4360.

[129] R. Huang, H. Cheng, Q. Chen, T. Huu-Toan, and X. Lin, Interactive learning for sensitivity factors of a human-powered augmentation lower exoskeleton, Proc. IEEE/RSJ Conf. on Intelligent Robots and Systems, Hamburg, Germany, 2015, 6409-6415.

[130] H. van der Kooij, B. Koopman, and E.H.F. van Asseldonk, Body weight support by virtual model control of an 
impedance controlled exoskeleton (LOPES) for gait training, Proc. IEEE Conf. on Engineering in Medicine and Biology Society, Vancouver, BC, Canada, 2008, 1969-1972.

[131] O. Unluhisarcikli, M. Pietrusinski, B. Weinberg, P. Bonato, and C. Mavroidis, Design and control of a robotic lower extremity exoskeleton for gait rehabilitation, Proc. IEEE/RSJ Conf. on Intelligent Robots and Systems, San Francisco, CA, USA, 2011, 4893-4898.

[132] K.N. Winfree, P. Stegall, and S.K. Agrawal, Design of a minimally constraining, passively supported gait training exoskeleton: ALEX II, Proc. IEEE Conf. on Rehabilitation Robotics, Zurich, Switzerland, 2011, 5975499.

[133] D. Zanotto, T. Lenzi, P. Stegall, and S.K. Agrawal, Improving transparency of powered exoskeletons using force/torque sensors on the supporting cuffs, Proc. IEEE Conf. on Rehabilitation Robotics, Seattle, WA, USA, 2013, 1-6.

[134] T. Lenzi, D. Zanotto, P. Stegall, M.C. Carrozza, and S.K. Agrawal, Reducing muscle effort in walking through powered exoskeletons, Proc. IEEE Conf. on Engineering in Medicine and Biology Society, San Diego, CA, USA, 2012, 3926-3929.

[135] G. Aguirre-Ollinger, Learning muscle activation patterns via nonlinear oscillators: application to lower-limb assistance, Proc. IEEE/RSJ Conf. on Intelligent Robots and Systems, Tokyo, Japan, 2013, 1182-1189.

[136] D. Zanotto, P. Stegall, and S.K. Agrawal, Adaptive assist-asneeded controller to improve gait symmetry in robot-assisted gait training, Proc. IEEE Conf. on Robotics and Automation, Hong Kong, China, 2014, 724-729.

[137] T. Petric, A. Gams, T. Debevec, L. Zlajpah, and J. Babic, Control approaches for robotic knee exoskeleton and their effects on human motion, Advanced Robotics, 27(13), 2013, 993-1002.

[138] W. van-Dijk, H. van-der-Kooij, B. Koopman, and E.H.F. van Asseldonk, Improving the transparency of a rehabilitation robot by exploiting the cyclic behaviour of walking, Proc. IEEE Conf. on Rehabilitation Robotics, Seattle, WA, USA, 2013, 1-8.

[139] J. Kerestes, T.G. Sugar, and M. Holgate, Adding and subtracting energy to body motion - Phase oscillator, Proc. ASME Conf. Design Engineering Technical and Computers and Information in Engineering, Buffalo, New York, USA, 2014, V05AT08A004.

[140] K. Seo, J. Lee, Y. Lee, T. Ha, and Y. Shim, Fully autonomous hip exoskeleton saves metabolic cost of walking, Proc. IEEE Conf. on Robotics and Automation, Stockholm, Sweden, 2016, 4628-4635.

[141] J. Olivier, A. Ortlieb, M. Bouri, and H. Bleuler, Influence of an assistive hip orthosis on gait, in Advances in Intelligent Systems and Computing, vol. 540 (Cham: Springer, 2017), $531-540$

[142] T.G. Sugar, E. Fernandez, D. Kinney, K.W. Hollander, and S. Redkar, HeSA, hip exoskeleton for superior assistance, in Wearable Robotics: Challenges and Trends, Biosystems and Biorobotics, vol. 16 (Cham: Springer, 2017), 319-323.

[143] W. van-Dijk, C. Meijneke, and H. van-der-Kooij, Evaluation of the achilles ankle exoskeleton, IEEE Transactions on Neural Systems and Rehabilitation Engineering, 25(2), 2017, 151-160.

[144] V.R. Garate, A. Parri, T. Yan, M. Munih, R.M. Lova, N. Vitiello, and R. Ronsse, Experimental validation of motor primitive-based control for leg exoskeletons during continuous multi-locomotion tasks, Frontiers in Neurorobotics, 11, 2017, $1-17$.

[145] A. Parri, T. Yan, F. Giovacchini, M. Cortese, M. Muscolo, M. Fantozzi, R.M. Lova, and N. Vitiello, A portable active pelvis orthosis for ambulatory movement assistance, in Wearable Robotics: Challenges and Trends, Biosystems and Biorobotics, vol. 16 (Cham: Springer, 2017), 75-80.

[146] K. Kasaoka and Y. Sankai, Predictive control estimating operator's intention for stepping-up motion by exo-skeleton type power assist system HAL, Proc. IEEE/RSJ Conf. Intelligent Robots and Systems, 2001, 1578-1583.

[147] J.L. Contreras-Vidal and R.G. Grossman, NeuroRex: a clinical neural interface roadmap for EEG-based brain machine interfaces to a lower body robotic exoskeleton, Proc.
IEEE Conf. on Engineering in Medicine and Biology, 2013, 1579-1582.

[148] K. Lee, D. Liu, L. Perroud, R. Chavarriaga, and J.D.R. Millan, Endogenous control of powered lower-limb exoskeleton, in Wearable Robotics: Challenges and Trends, Biosystems and Biorobotics, vol. 16 (Cham: Springer, 2017), 115-119.

[149] K. Gui, H. Liu, and D. Zhang, Toward multimodal humanrobot interaction to enhance active participation of users in gait rehabilitation, IEEE Transactions on Neural Systems and Rehabilitation Engineering, 25(11), 2017, 2054-2066.

[150] W. Meng, B. Ding, Z. Zhou, Q. Liu, and Q. Ai, An EMG-based force prediction and control approach for robotassisted lower limb rehabilitation, Proc. IEEE Conf. on Systems, Man and Cybernetics, San Diego, CA, USA, 2014, 2198-2203.

[151] W. Meng, Y. Zhu, Z. Zhou, K. Chen, and Q. Ai, Active interaction control of a rehabilitation robot based on motion recognition and adaptive impedance control, Proc. IEEE Conf. on Fuzzy Systems, Beijing, China, 2014, 1436-1441.

[152] Y. Fan, Z. Guo, and Y. Yin, sEMG-based neuro-fuzzy controller for a parallel ankle exoskeleton with proprioception, International Journal of Robotics and Automation, 26(4), 2011, 450-460.

[153] S.-H. Lee, S.-N. Yu, H.-D. Lee, S.-J. Hong, C.-S. Han, and J.-S. Han, Proposal for a modular-type knee-assistive wearable unit and verification of its feasibility, Int. Symposium on Automation and Robotics in Construction, 2008, 187-194.

[154] H. Kawamoto and Y. Sankai, Power assist system HAL-3 for gait disorder person, in Lecture Notes in Computer Science vol. 2398 (Berlin, Heidelberg: Springer, 2002), 196-203.

[155] T. Kawabata, H. Satoh, and Y. Sankai, Working posture control of robot suit HAL for reducing structural stress, Proc. IEEE Conf. on Robotics and Biomimetics, Guilin, China, 2009, 2013-2018.

[156] C. Fleischer, A. Wege, K. Kondak, and G. Hommel, Application of EMG signals for controlling exoskeleton robots, Biomedizinische Technik, 51(5-6), 2006, 314-319.

[157] H. He and K. Kiguchi, A study on EMG-based control of exoskeleton robots for human lower-limb motion assist, Int. Special Topic Conf. on Information Technology Applications in Biomedicine, Tokyo, Japan, 2007, 292-295.

[158] K. Kiguchi and Y. Imada, EMG-based control for lowerlimb power-assist exoskeletons, IEEE Workshop on Robotic Intelligence in Informationally Structured Space, Nashville, TN, USA, 2009, 19-24.

[159] Y. Hayashi and K. Kiguchi, A lower-limb power-assist robot with perception-assist, Proc. IEEE Conf. on Rehabilitation Robotics, Zurich, Switzerland, 2011, 5975445.

[160] Y. Hayashi and K. Kiguchi, Stairs-ascending/descending assist for a lower-limb power-assist robot considering ZMP, Proc. IEEE/RSJ Conf. on Intelligent Robots and Systems, San Francisco, CA, USA, 2011, 1755-1760.

[161] K. Kiguchi, A. Komori, and T. Kouno, A study on motion modification force in perception-assist for a lower-limb powerassist exoskeleton, Proc. Int. Symposium on Soft Computing and Intelligent Systems, Kitakyushu, Japan, 2014, 1233-1237.

[162] K. Kiguchi and Y. Yokomine, Perception-assist with a lowerlimb power-assist robot for sitting motion, Proc. IEEE Conf. on Systems Man and Cybernetics, Kowloon, China, 2015, 2390-2394.

[163] Y. Chen, J. Hu, W. Wang, L. Peng, L. Peng, and Z.-G. Hou, An FES-assisted training strategy combined with impedance control for a lower limb rehabilitation robot, Proc. IEEE Conf. on Robotics and Biomimetics, 2013, 2037-2042.

[164] Y. Fan and Y. Yin, Active and progressive exoskeleton rehabilitation using multisource information fusion from EMG and force-position EPP, IEEE Transactions on Biomedical Engineering, 60(12), 2013, 3314-3321.

[165] L. Grazi, S. Crea, A. Parri, T. Yan, M. Cortese, F. Giovacchini, M. Cempini, G. Pasquini, S. Micera, and N. Vitiello, Gastrocnemius myoelectric control of a robotic hip exoskeleton, Proc. IEEE Conf. on Engineering in Medicine and Biology Society, Milan, Italy, 2015, 3881-3884.

[166] J.R. Koller, D.A. Jacobs, D.P. Ferris, and C.D. Remy, Learning to walk with an adaptive gain proportional myoelectric 
controller for a robotic ankle exoskeleton, Journal of Neuroengineering and Rehabilitation, 12, 2015, 97.

[167] A. Shabani and M.J. Mahjoob, Bio-signal interface for knee rehabilitation robot utilizing EMG signals of thigh muscles, Proc. Int. Conf. on Robotics and Mechatronics, Tehran, Iran, 2016, 228-233.

[168] C. Mavroidis, J. Nikitczuk, B. Weinberg, R. Arango, G. Danaher, K. Jensen, M. Leahey, R. Pavone, P. Pelletier, A. Provo, J. Prugnarola, R. Stuart, and D. Yasevac, Smart portable rehabilitation devices, Journal of NeuroEngineering and Rehabilitation, 2(1), 2005, 18

[169] H. Kawamoto, S. Taal, H. Niniss, T. Hayashi, K. Kamibayashi, K. Eguchi, and Y. Sankai, Voluntary motion support control of robot suit HAL triggered by bioelectrical signal for hemiplegia, Proc. IEEE Conf. on Engineering in Medicine and Biology Society, Buenos Aires, Argentina, 2010, 462-466.

[170] H.-Y. Huang, J.-S. Chen, and C.-E. Huang, Toward the gait analysis and control of a powered lower limb orthosis in ascending and descending stairs, Procedia Engineering, 79, $2014,417-426$.

[171] N. Karavas, A. Ajoudani, N. Tsagarakis, J. Saglia, A. Bicchi, and D. Caldwell, Tele-impedance based assistive control for a compliant knee exoskeleton, Robotics and Autonomous Systems, 73, 2015, 78-90.

\section{Biographies}

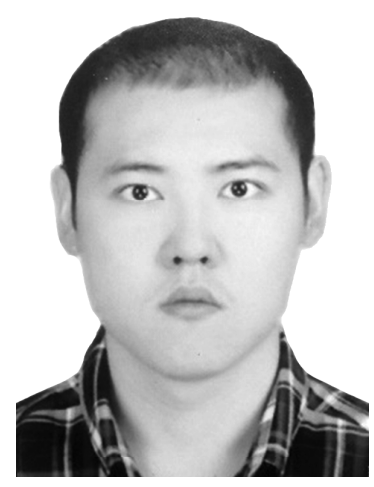

and exoskeleton robot.

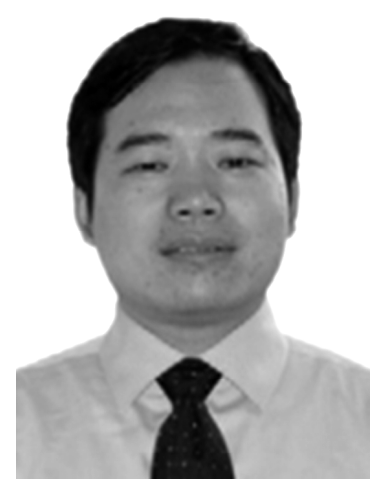

Xinyu $W u$ is now a Professor at Shenzhen Institutes of Advanced Technology, and the Director of Center for Intelligent Bionic. He received his B.E. and M.E. degrees from the Department of Automation, University of Science and Technology of China in 2001 and 2004, respectively. His Ph.D. degree was awarded at the Chinese University of Hong Kong in 2008. He has published over 180 papers and two monographs. His research interests include computer vision, robotics and intelligent system.

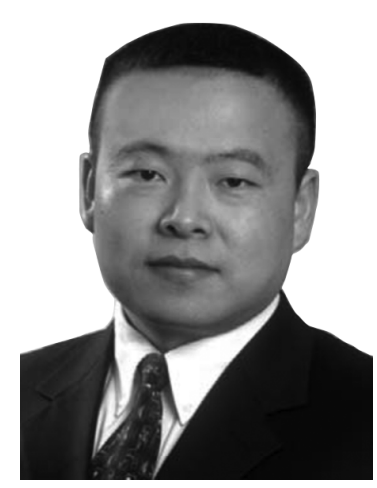

Jingang Yi (S'99-M'02-SM'07) received the B.S. degree in electrical engineering from Zhejiang University, Hangzhou, China, in 1993, the M.Eng. degree in precision instruments from Tsinghua University, Beijing, China, in 1996, and the M.A. degree in mathematics and the Ph.D. degree in mechanical engineering from the University of California, Berkeley, in 2001 and 2002, respectively. From May 2002 to January 2005, he was with Lam Research Corporation, Fremont, CA, as a Member of the Technical Staff. From January 2005 to December 2006, he was with the Department of Mechanical Engineering, Texas, A\&M University, College Station, as a Visiting Assistant Professor. From January 2007 to July 2008, he was an Assistant Professor of mechanical engineering with San Diego State University, San Diego, CA. In August 2008, he joined Rutgers University, Piscataway, NJ, where he is currently an Assistant Professor of mechanical engineering. His current research interests include autonomous robotic systems, dynamic systems and control, intelligent sensing and actuation systems, mechatronics, and automation science and engineering, with applications to semiconductor manufacturing, intelligent transportation, and biomedical systems. He is a member of American Society of Mechanical Engineers (ASME). He is a member of the Conference Editorial Board of the IEEE Robotics and Automation Society and ASME Dynamic Systems and Control Division. He is a Guest Editor of the IEEE Transactions on Automation Science and Engineering.

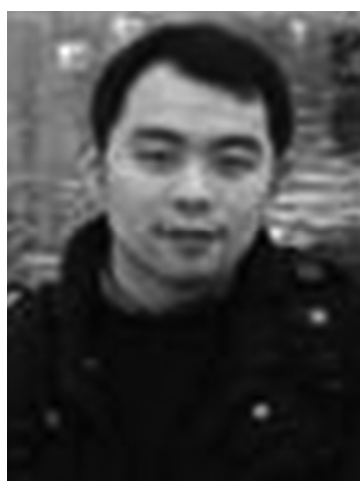

Can Wang received his bachelor degree from Zhejiang University in 2001, and his Ph.D. degree in multirobot coordination from the City University of Hong Kong in 2009. He was a post-doc researcher in Lab GREYC of CNRS, France. $\mathrm{He}$ is now an Associate Professor with the Center for Intelligent and Biomimetic Systems at Shenzhen Institutes of Advanced Technology, Chinese Academy of Sciences. His current research interests include networked mobile robots, artificial intelligence, exoskeleton robot and micromanipulation of bio-cells.

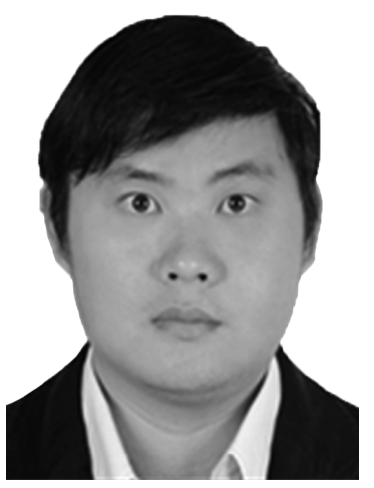

Chunjie Chen received his B.E. and M.E. degrees from Wuhan University and received his Ph.D degree from the Chinese Academy of Sciences. In recent 5 years, he has over 15 projects supported by the Natural Science Foundation of China, the National Key Research and Development Program of China, etc. He has published over 20 papers in international robotic journals and conferences. He has obtained over 15 national patents. His main research interests include exoskeleton robot, service robots and intelligent system. 